3. Peter J. Cameron, Peter M. Neumann and Jan Saxl, 'An interchange property in finite permutation groups', Bull. London Math. Soc., 11 (1979), 161-169.

. Minoru Numata, 'Generalization of a theorem of Peter J. Cameron', Osaka J. Math., 15 (1978), 311-342.

5. Jan Saxl, 'On points and triples in Steiner triple systems', to appear.

6. M. S. Smith, 'On rank 3 permutation groups', J. Algebra 33 (1975), 22-42.

Merton College

Oxford

New Hall

Cambridge

\section{ON TWINS IN THE FOUR-SPHERE I}

\section{By JOSÉ M. MONTESINOS*}

[Received 9th November 1981]

\section{Introduction}

IN $[\mathbf{Z}]$, Zeeman introduced the process of twist-spinning a 1-knot to obtain a 2-knot, and proved that a twist-spun knot is fibered with structure group finite cyclic.

In [L], Litherland generalized twist-spinning by performing during the spinning process the "rolling" operation, introduced by Fox [F] and defined rigorously by Litherland [L]. He showed that, provided one combines the rolling with a twist, the resulting knot is fibered. However the bundle structure group need not to be finite.

In this paper we generalize those results by introducing the concept of twin. A twin $W$ is a subset of $S^{4}$ made of two 2-knots $R$ and $S$ which intersects transversally in two points (Section 3 ). The prototype of a twin is the $n$-twist spun twin of $K$, i.e. the union of the $n$-twist spun knot of $K$ and the boundary of the 3-ball in which the original knotted arc $K$ lies.

The exterior of a twin, $X(W)$, is the closure of $S^{4}-N(W)$, where $N(W)$ is a regular neighbourhood of $W$ in $S^{4}$. It turns to be that the 0 -twist spun twin of $K$ and the $2 k$-twist spun twin of $K$ both have the same exterior (and the same regular neighbourhood). In fact, in Section 1 we will prove that the circular deformation of Figure 1.3 (which takes place in $N(W)$ ) is isotopic to the 2-twist spun deformation. Intuitively, by pulling the two free ends of the fifth picture of Figure 1.3 the knot would turn around twice! This is the main reason for introducing the concept of twin.

The above observation makes it very interesting to investigate Dehnsurgery on a twin, i.e. the result of replacing $N(W)$ by itself using some gluing automorphism $\varphi$ of $\partial N(W)$. Here $\partial N(W)$ is the tridimensional torus and the pair $\left(S^{4}, W\right)$ is completely determined by $X(W)$ and the (framed) meridians of the components $R$ and $S$ of the twin (Section 3 ).

To study Dehn-surgery on $W$ we first characterize those automorphisms $\varphi$ of $\partial N(W)$ which extend to $N(W)$. The answer, given in Section 5 is the following. First, $\varphi$ must fix the meridian torus $T$, i.e. the 2-torus where $\partial N(R)$ and $\partial N(S)$ intersect. And second, if the meridians of $R$ and $S$ are thought of as a basis $b$ of $T$, then $b$ and $\varphi(b)$ must be related by an even matrix, i.e. an integer matrix in which the sum of the entries is even.

* Supported by "Comisión Asesora del Ministerio de Educación."

Quart. J. Math. Oxford (2), 34 (1983), 171-199 
This rather curious result is used to characterize the automorphisms of a 2-torus standarly embedded in $S^{4}$, which extend to $S^{4}$, and also to prove that any homotopy sphere gotten by Dehn-surgery on such a 2-torus is the real $S^{4}$.

The set of even matrices is an abnormal subgroup of $\operatorname{SL}(2, \mathbb{Z})$ of index three (Section 4). Hence if $\varphi$ is an automorphism of $\partial N(W)$ which fixes $T$, we obtain three possible homotopy 3-spheres by performing Dehnsurgery using $\varphi$. Of course, if $b$ and $\varphi(b)$ are related by an even matrix the corresponding homotopy sphere is $S^{4}$. The other two are $R^{*}$ and $S^{*}$, i.e. the manifolds obtained by Gluck-surgery on $R$ and $S$ (Section 8). Furthermore, the twins $W$ and $W^{\prime}$ obtained by $\varphi$ such that $b$ and $\varphi(b)$ are related by an even matrix, have a remarkable relationship, since $W^{\prime}$ comes from $W$ by an alternative sequence of "2-spinnings" of $R$ around $S$ and of $S$ around $R$, to be determined by the matrix.

If the automorphism $\varphi$ of $N(W)$ does not fix $T$, we study some cases in which the resulting 4-manifold is $S^{4}$. The new twin $W^{\prime}$ is then the result of a sequence of 2 -spinnings in $W$ and a rolling operation which contains, in particular, the one defined by Litherland. Thus the 2-knots of Litherland are a particular case of Dehn-surgery.

The second part of this paper (to appear elsewhere) will be devoted to the fibration problem, i.e. given a twin in $S^{4}$, try to understand what surgeries in $W$ give a twin $W^{\prime}$ which has a component which is a fibered knot. As a particular case we will get the results of Zeeman and Litherland referred to above.

I want to thank Francisco González-Acuña for many enlightening conversations about the presentation of Section 4. I thank also Francis Bonahon for his helpful criticism in reading this paper.

Notation. It will appear clear when we are working in the smooth or $P L$ category. In this case all submanifolds are assumed to be locally flat.

$(m, n, p, \ldots)$ stands for the g.c.d. of the integers $m, n, p, \ldots$

$|A|$ stands for the determinant of the matrix [A]

$N(X)$ stands for a suitable regular neighbourhood of the set $X$.

\section{The initiating example}

Let $R$ be a 2 -sphere trivially embedded in $S^{4}$, i.e. $R$ bounds a 3-ball $B^{3}$ which is the page of an open-book structure of $S^{4}$ with binding $R$ and with monodromy the identity automorphism of $B^{3}$. Let $A$ be a knotted spanning arc in $B^{3}$. By rotating $B^{3}$ around $R$, the arc $A$ generates a $2-k n o t S$ which is called the spun knot of $A$. If during the rotation the arc A spins around itself $n$ times, it generates the 2-knot called the $n$-twist spun knot of $A$ (see $[\mathbf{Z}]$ ).
A different description of those knots is the following. Think of $S^{4}$ as $\partial\left(B^{2} \times B^{3}\right)=S^{1} \times B^{3} \cup B^{2} \times S^{2}$. Let $S^{0}$ be the poles of $S^{2}$ and assume $\partial A=S^{0}$. Then the spun knot of $A$ is the pair $\left(S^{1} \times B^{3} \cup B^{2} \times S^{2}, S^{1} \times A \cup\right.$ $\left.B^{2} \times S^{0}\right)$. Let $\tau$ be the automorphism of $S^{1} \times S^{2}$ defined by $\tau(\theta, x)=$ $(\theta, \rho(\theta) x)$, where $\rho(\theta)$ is the angle $\theta$ rotation of $S^{2}$ around the poles. Then the pair $\left(S^{1} \times B^{3} \cup_{i \pi^{n}} B^{2} \times S^{2}, S^{1} \times A \cup B^{2} \times\right.$ the $S^{0}$ ), where $i$ is the natural identification betwentity). To see this, note $S^{1} \times B^{3}$ and $B^{2} \times S^{2}$ (note that $\tau \mid S^{1} \times S^{0}$ is the identity). To see the note that $\tau$ extends naturally to an automorphism $\bar{\tau}$ of $S^{1} \times B^{3}$. Then, under the homeomorphism $\left(\bar{\tau}^{n} \cup i d\right): S^{1} \times B^{3} \cup_{i \pi^{n}} B^{2} \times S^{2} \rightarrow S^{1} \times B^{3} \cup B^{2} \times S^{2}$, the set $S^{1} \times A \cup B^{2} \times S^{0}$ is mapped to the $n$-twist spun knot of $A$ as defined above.

For $n=2 k$ we have another description of the $2 k$-twist spun knot of $A$, based on the fact that $\tau^{2 k}$ extends to $B^{2} \times S^{2}$. This is the starting point of the example which originated the study made in this paper. Let us consider, for the sake of simplicity, the case $n=2$.

The extension of $\tau^{2}$ to $B^{2} \times S^{2}$ depends on the existence of an isotopy F: $S^{1} \times S^{2} \times[0,1] \rightarrow S^{1} \times S^{2} \times[0,1]$ from $\tau^{2}$ to the identity [GL]. Thus

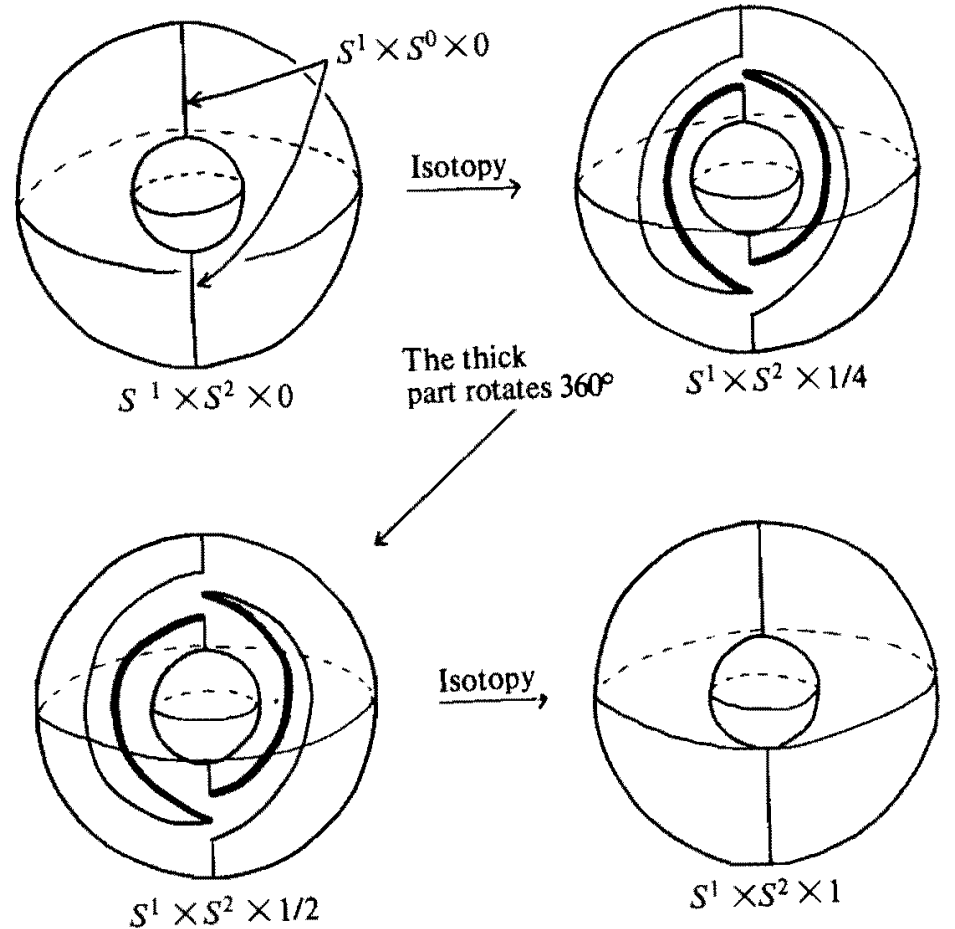

Fro. 1.1 


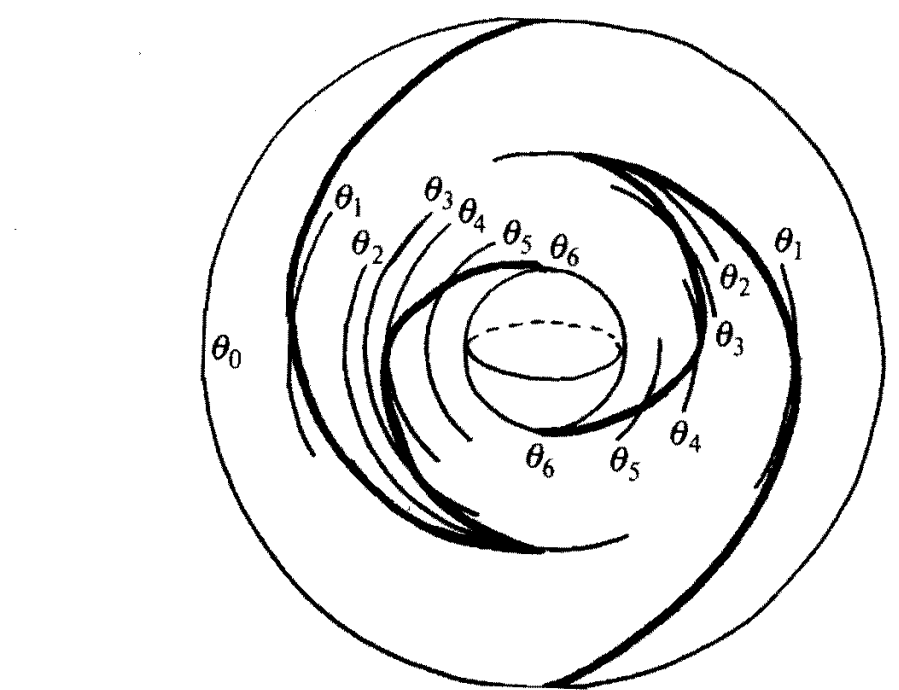

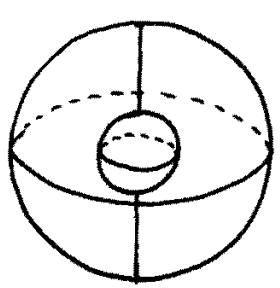

$\left\{\theta_{0} \times S^{2} \times[0,1]\right\}$

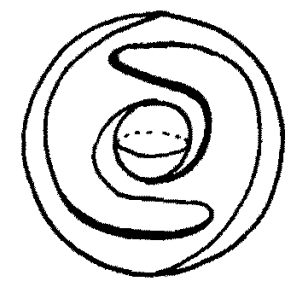

$\theta_{3} \times S^{2} \times[0,1]$
Detail of $S^{1} \times S^{2} \times 1 / 4$

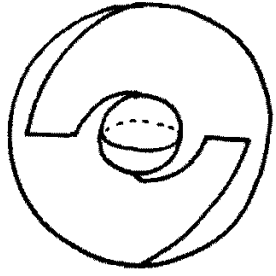

$\theta_{1} \times S^{2} \times[0,1]$

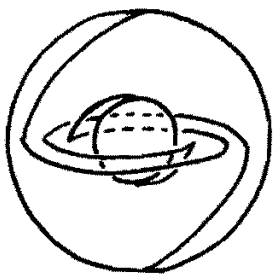

$\theta_{4} \times S^{2} \times[0,1]$

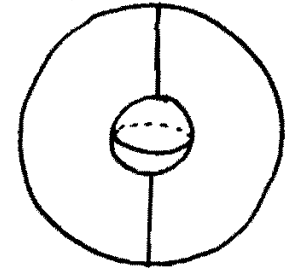

$\theta_{6} \times S^{2} \times[0,1]$

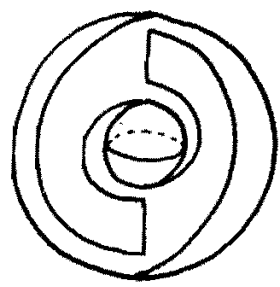

$\theta_{2} \times S^{2} \times[0,1]$

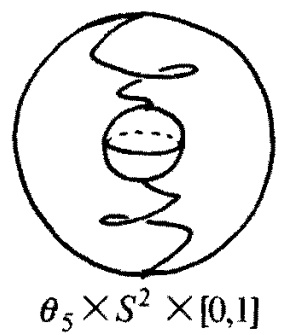

FiG. 1.2 identifying $S^{1} \times S^{2} \times[0,1]$ with a collar of $\partial\left(B^{2} \times S^{2}\right)$ in $B^{2} \times S^{2}, F$ extends to an automorphism $G$ of $B^{2} \times S^{2}$ which is $\tau^{2}$ in the boundary. Hence, under the homeomorphism $\psi=(i d \cup G)$ from $S^{1} \times B^{3} \cup_{i \tau^{2}} B^{2} \times S^{2}$ onto $S^{1} \times B^{3} \cup B^{2} \times S^{2}=S^{4}$, the set $S^{1} \times A \cup B^{2} \times S^{0}$ is mapped to the 2-twist spun knot of $A$.

We want to visualize the image $\psi\left(S^{1} \times A \cup B^{2} \times S^{0}\right)$ in $S^{4}$. The intersection of $S^{1} \times A \cup B^{2} \times S^{0}$ with the collar $S^{1} \times S^{2} \times[0,1]$ is $S^{1} \times S^{0} \times[0,1]$. But $\psi$ is the identity outside of that collar. Hence we only have to visualize the effect of $F$ in the pair $\left(S^{1} \times S^{2} \times[0,1], S^{1} \times S^{0} \times[0,1]\right)$. For this, we use the "movie method".

The isotopy $F$ appears in Figure 1.1 (compare [GL]), in which the time parameter is $t \in[0,1]$. Now, we obtain the "circular movie" of $F$ in Figure 1.2 in which the time parameter is $\theta \in S^{1}$. The top picture of Figure 1.2 shows a relevant step of Figure 1.1 with some of the levels $\theta_{i} \times S^{2} \times \frac{1}{4}$ marked; and this picture helps to obtain the rest of Figure 1.2. Finally, to visualize the total effect of the map $\psi$ we add to the circular movie of Figure 1.2, which takes place in a collar of $\partial\left(B^{2} \times S^{2}\right)$, the rest of $S^{4}$ in which $\psi$ is the identity. For the arc $A$ being a "trefoil are" the total effect of $\psi$ is shown in Figure 1.3.

Thus, what Figure 1.3 is telling us is that the usual movie description of the $2 k$-twist spun knot as an arc spinning around itself $2 k$ times while it spuns around $R$, can be isotoped so that it fixes a small ball containing the "knot". In other words, the two sets $R \cup S$, and $R \cup(2 k$-twist spun knot of $A$ ) have the same exterior (and the same regular neighbourhood $E^{4}$ ). Thus the $2 k$-spinning process is independent of the ambient space in which $E^{4}$ lies (as the usual movie picture of the 2-knot falselly suggest). On the contrary, that process can be defined only in terms of the triple $\left(E^{4}, R, S\right)$ which we call twin in the next section.

Hence the first part of this paper will deal with an "abstract" study of the triple $(E, R, S)$, concentrating in the problem of what automorphisms of $\partial E$ extend to $E$. After that we will study the problem of performing
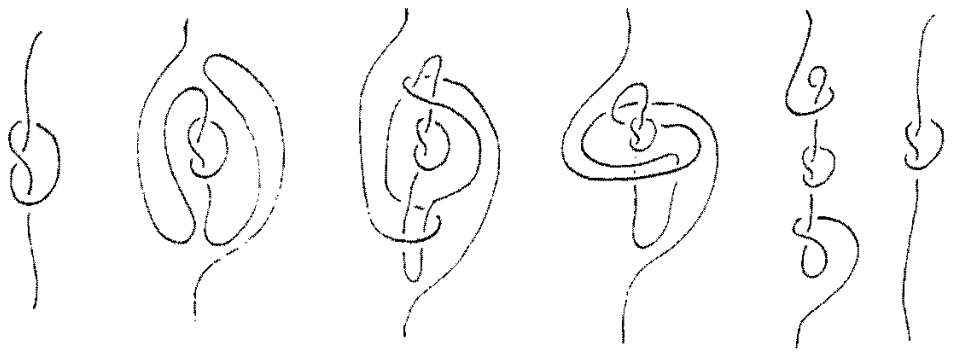

FIO. 1.3 
surgery on a "concrete" twin lying in $S^{4}$ so that we get new twins (and also new knots) in a homotopy 4 -sphere. Then we use our understanding about automorphisms of $\partial E$ which extend to $E$, to recognize those as the real $S^{4}$ in some cases.

\section{Twins}

Represent $S^{1}$ as the quotient $\mathbb{R} / \mathbb{Z}$ and denote by $T^{3}$ the tridimensional torus $S^{1} \times S^{1} \times S^{1}$. Each vector $\left(a_{1}, a_{2}, a_{3}\right) \in \mathbb{R}^{3}$ defines an oriented straight line in $\mathbb{R}^{3}$ and consequently an oriented simple curve in $T^{3}$ which is closed if and only if the ratios $a_{i} / a_{j}$ are rational. Any oriented simple closed curve in $T^{3}$ which is isotopic to a curve defined by some vector $\left(a_{1}, a_{2}, a_{3}\right)$ will be called canonical. Let $l, r, s$ be the canonical curves in $T^{3}$ defined by the vectors $(1,0,0),(0,1,0),(0,0,1)$, respectively. These curves, of course, represent a basis of $H_{1}\left(T^{3} ; \mathbb{Z}\right)=\mathbb{Z} \oplus \mathbb{Z} \oplus \mathbb{Z}$.

Let $\alpha$ be a canonical curve in $T^{3}$. Then $\alpha$ is isotopic to a curve of vector $\vec{a}=\left(a_{1}, a_{2}, a_{3}\right)$, where $a_{1}, a_{2}, a_{3}$ are relatively prime integers. Thus, $\alpha$ is homologous to $a_{1} l+a_{2} r+a_{3} s$ in $T^{3}$. Now it is possible to find integer vectors $\vec{b}=\left(b_{1}, b_{2}, b_{3}\right)$ and $\vec{c}=\left(c_{1}, c_{2}, c_{3}\right)$ such that the volume of the parallelepiped generated by $\vec{a}, \vec{b}$ and $\vec{c}$ is 1 (see, for instance, [NW] p. 15 , Corollary II.1). In other words, the linear transformation $\psi: \mathbb{R}^{3} \rightarrow \mathbb{R}^{3}$ with equations

$$
\varphi\left(x_{1}, x_{2}, x_{3}\right)=\left(x_{1}, x_{2}, x_{3}\right)\left[\begin{array}{lll}
a_{1} & a_{2} & a_{3} \\
b_{1} & b_{2} & b_{3} \\
c_{1} & c_{2} & c_{3}
\end{array}\right]
$$

induces an automorphism of $T^{3}$ which sends $l$ to $\alpha$. Thus a canonical curve is nothing else than the image of $l$ by some automorphism $\varphi$ of $T^{3}$, since that $\varphi$ must be isotopic to a linear one.

Let $\{*\}$ be a point close to $(0,0,0)$ in the $y, z$ plane of $\mathbb{R}^{3}$. Then $\mathbb{R} \times\{*\} \subset \mathbb{R}^{3}$ defines a framing of $l$ and, via $\varphi$, a framing of $\varphi(l)=\alpha$. Clearly, this framing of $\alpha$ does not depend on the particular $\varphi$, and it is called the natural framing of $\alpha$.

Remark 1.1. If $\alpha$ is a canonical curve in $T^{3}$, the natural framing can be characterized as the unique framing such that the corresponding Milnorsurgery gives $S^{1} \times S^{2} \# S^{1} \times S^{2}$. It is natural to conjecture that a simple closed curve in $T^{3}$ is canonical if and only if some Milnor-surgery on it gives $S^{1} \times S^{2} \# S^{1} \times S^{2}$.

In $S^{4}=\mathbb{R}^{4}+\{\infty\}$ take the set $E^{4}=\left\{\left(x_{1}, x_{2}, x_{3}, x_{4}\right) \in \mathbb{R}^{4} \mid x_{1}^{2}+x_{2}^{2} \leqslant 1\right.$ or $\left.x_{3}^{2}+x_{4}^{2} \leqslant 1\right\} \cup\{\infty\}$. Thus $E^{4}$ is a regular neighbourhood of the union of the coordinate planes $R=\mathbb{R}^{2} \times\{0\} \cup\{\infty\}$ and $S=\{0\} \times \mathbb{R}^{2} \cup\{\infty\}$, which meet transversally in $\{0\}$ and $\{\infty\}$.
We call twin the triple $\left(E^{4}, R, S\right)$. The name was suggested by two twin cristals ("macla" in spanish). Thus a twin is a sort of plumbing of two 2-spheres.

The complement $S^{4}$-int $E^{4}$ is a regular neighbourhood of the torus

$$
F^{2}=\left\{\left(x_{1}, x_{2}, x_{3}, x_{4}\right) \in \mathbb{R}^{4} \mid x_{1}^{2}+x_{2}^{2}=2=x_{3}^{2}+x_{4}^{2}\right\} .
$$

Hence $\partial E^{4}$ is homeomorphic to $T^{3}$. An explicit homeomorphism is $\varphi: T^{3} \rightarrow \partial E^{4}$ given by

$(\lambda, \theta, \mu) \mapsto\left\{\begin{array}{c}((\tan \pi \lambda+1) \cos 2 \pi \theta,(\tan \pi \lambda+1) \sin 2 \pi \theta, \\ \cos 2 \pi \mu, \sin 2 \pi \mu), \quad 0 \leqslant \lambda \leqslant \frac{1}{2} \\ (\cos 2 \pi \theta, \sin 2 \pi \theta,(\tan \pi(1-\lambda)+1) \cos 2 \pi \mu, \\ (\tan \pi(1-\lambda)+1) \sin 2 \pi \mu),\end{array}\right.$

We identify $\partial E^{4}$ with $T^{3}$ by $\varphi$. A meridian of $R$ (resp. $S$ ) is a framed simple closed curve in $T^{3}$ such that it bounds a framed disc properly embedded in $E^{4}$ which cuts $R$ (resp. $S$ ) transversally in one point. For instance $r$ and $s$ (with the canonical framings) are meridians of $R$ and $S$ respectively. A meridian torus is a 2-torus embedded in $T^{3}$ which contains a system of meridians of the twin, i.e. a basis made up of meridians of $R$ and $S$. For instance the torus $T^{2}$ defined by the equation $\lambda=0$ is a meridian torus.

\section{The structure of a twin}

First we describe a handle presentation of $E^{4}$ (refer to Diagram 3.1), Let $D^{4}$ be a regular neighbourhood in $E^{4}$ of a simple arc in $S$

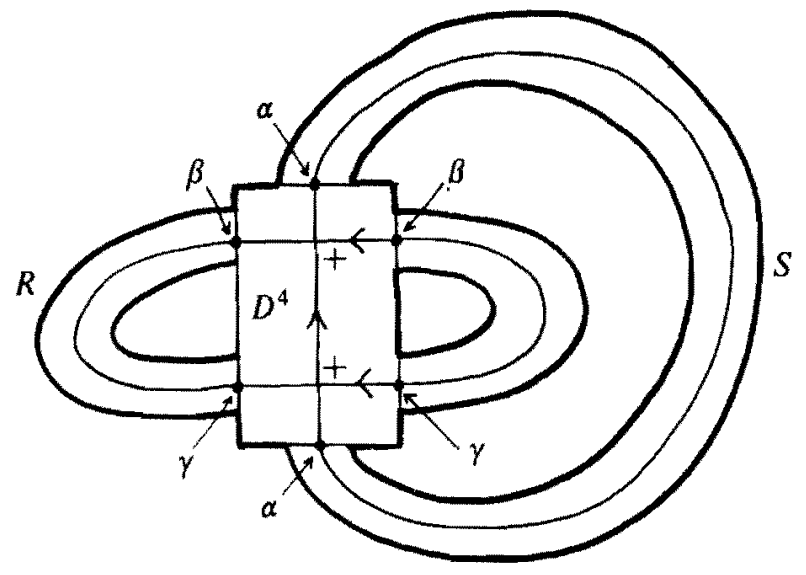

FIG. 3.1

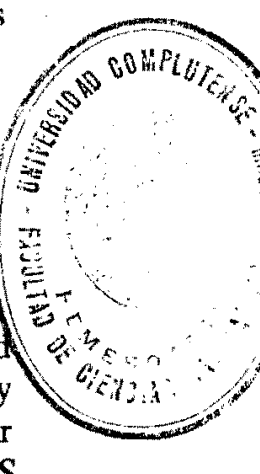




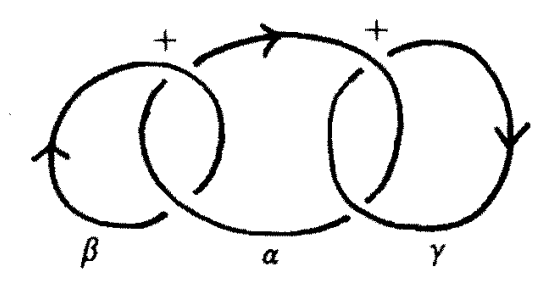

Fio. 3.2

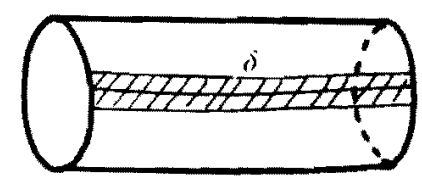

$S^{1} \times[0,1]$

FIG. 3.3

connecting the points of $R \cap S$. The curves $\alpha, \beta, \gamma$ in $\partial D^{4}=\mathbb{R}^{4}+\{\infty\}$ are shown in Figure 3.2.

We now add a 2 -handle to $D^{4}$ along $\alpha$ with framing zero to complete a regular neighbourhood of $S$. Finally add a thickened tube $\left(S^{1} \times[0,1]\right) \times$ $D^{2}$ to $D^{4}$ along $\beta \cup \gamma$ to complete a regular neighbourhood of $R$. This is done in two steps (see Figure 3.3).

In the first place we paste $B^{1} \times[0,1] \times D^{2}$ to $D^{4}$, where $B^{1}$ is a 1 -disc embedded in $S^{1}$. This is the same as adding a 1-handle to $D^{4}$ (we must take care that the orientations induced in $\beta, \gamma$ by the tube be different). The rest of the tube is added by attaching a 2 -handle along $\beta^{1} \cup \gamma^{1}$ (see Figure 3.4).

Thus $E^{4}$ is composed by one 0 -handle, one 1-handle and two 2handles. In Figure 3.4 we have shadowed the parts of $R$ and $S$ lying in $D^{4}$. We have also indicated the meridians $r, s$ of $R, S$, respectively.

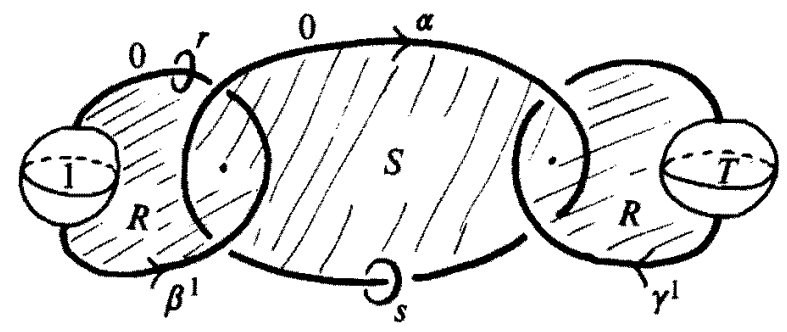

FiG. 3.4

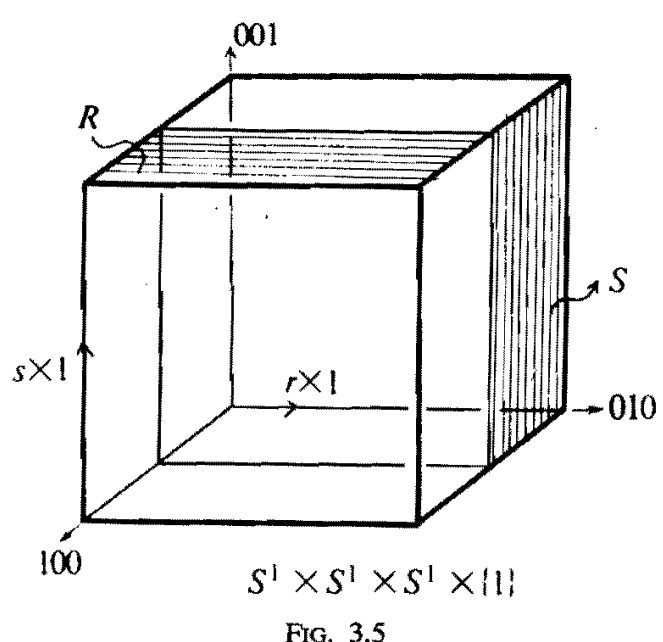

Now we describe the dual handle representation. We start with $T^{3} \times$ $[0,1]$ and we add two 2-handles along the framed $r \times\{1\}$ and $s \times\{1\}$ (with the natural framings). Afterwards we fill it up with a copy of $S^{1} \times B^{3}$ (i.e. 3-handle plus 4-handle). The two 2-handles are regular neighbourhoods of the meridian discs of $R$ and $S$. These regular neighbourhoods have a natural product structure which agree with the natural framings of $r$ and $s$.

This last description leads to a very convenient way of visualizing a twin. Take $T^{3} \times[0,1]$ and define an identification on $T^{3} \times\{1\}$ as follows. The lines of equations $\{x=a, z=b\}$, where $a \equiv a^{\prime}(\bmod \mathbb{Z}), a^{\prime} \in\left[\frac{1}{2}, 1\right]$; or $\{x=a, y=b\}$, where $a \equiv a^{\prime}(\bmod \mathbb{Z}), a^{\prime} \in\left[0, \frac{1}{2}\right]$, collapse to points. The result of the identifications is $E^{4}$. The image by the identification of the first (resp. second) system of lines is $R$ (resp. $S$ ) (see Figure 3.5).

\section{Disgression: A certain subgroup of $\operatorname{OL}(2, \mathbb{Z})$}

Let $M$ be a closed, oriented, smooth 4-manifold with $H_{1}(M ; \mathbb{Z} / 2 \mathbb{Z})=0$, and let $C^{2}$ be a closed, orientable 2 -manifold which represents an element of $H_{2}(M ; \mathbb{Z} / 2 \mathbb{Z})$ which is Poincaré-dual of $w_{2}(M)$. A membrane $P$ for $C$ is a compact surface embedded in $M$, normal to $C$ along its boundary (contained in $C$ ) and such that its interior is transverse to $C$. Move $\boldsymbol{P}$ to get $\boldsymbol{P}^{\prime}$, transverse to $\boldsymbol{P}$, in such a way that $\boldsymbol{P}$ slides parallel to itself over $C$. The number of points in Int $P \cap\left(P^{\prime} \cup C\right)$, reduced $\bmod 2$, only depends on the homology class of $\partial P$ in $H_{1}(C ; \mathbb{Z} / 2 \mathbb{Z})$ and this defines a quadratic form $q: H_{1}(C ; \mathbb{Z} / 2 \mathbb{Z}) \rightarrow \mathbb{Z} / 2 \mathbb{Z}$ with respect to the homology intersection form on $C$. This form was introduced by Roklin in $[\mathbf{R}]$. 
Let $T^{2}$ be the 2-dimensional torus, and let $e: T^{2} \hookrightarrow S^{4}$ be an embedding. Then we have the Roklin quadratic form $q: H_{1}(T ; \mathbb{Z} / 2 \mathbb{Z}) \rightarrow \mathbb{Z} / 2 \mathbb{Z}$.

Denote by Aut $G$ the group of automorphisms of a group $G$. In view of the following proposition, we are interested in the subgroup $H$ of Aut $H_{1}(T ; \mathbb{Z})$ whose elements, reduced $\bmod 2$, are the $q$-isometries of $H_{1}(T ; \mathbb{Z} / 2 \mathbb{Z})$. This subgroup depends on the embedding $e$. But for a different embedding we obtain a group which is conjugate to $H$. In fact, the Arf invariant of $q$ is zero $[\mathbf{R} ; 4]$, and this determines the isomorphism class of $q$ [MH; appendix 1].

Propostrion 4.1. Let $\varphi$ be an automorphism of $T^{2}$. If $\varphi$ extends to an automorphism of $S^{4}$ then $\varphi_{*}: H_{1}\left(T^{2} ; \mathbb{Z}\right) \rightarrow H_{1}\left(T^{2} ; \mathbb{Z}\right)$ belongs to $H$.

Proof. It is a trivial consequence of the definition of $q$.

The fact that $q$ has Arf invariant zero $[\mathbf{R} ; 4]$ means that only one element of $H_{1}\left(T^{2} ; \mathbb{Z} / 2 \mathbb{Z}\right)$ is evaluated to $1 \in \mathbb{Z} / 2 \mathbb{Z}$ by $q$. The preimage of that element by the natural epimorphism $H_{1}\left(T^{2} ; \mathbb{Z}\right) \rightarrow H_{1}\left(T^{2} ; \mathbb{Z} / 2 \mathbb{Z}\right)$ is a lattice $L$ in $H_{1}\left(T^{2} ; \mathbb{Z}\right)$. Thus $H$ is the stabilizer of $L$ in Aut $H_{1}\left(T^{2} ; \mathbb{Z}\right)$.

Propostrion 4.2. $H$ is a subgroup of Aut $H_{1}\left(T^{2} ; \mathbb{Z}\right)$ of index 3 which is not nomal.

Proof. We have the epimorphism Aut $H_{1}\left(T^{2}, \mathbb{Z}\right) \rightarrow$ Aut $H_{1}\left(T^{2} ; \mathbb{Z} / 2 \mathbb{Z}\right) \cong$ Aut $(\mathbb{Z} / 2 \mathbb{Z} \oplus \mathbb{Z} / 2 \mathbb{Z})=\sigma_{3}$, where $\sigma_{3}$ is the symmetric group of 3 indices Then, $H$ is conjugate to the preimage of the stabilizer of any index.

Let $r, s$ be a basis of $H_{1}\left(T^{2} ; \mathbb{Z}\right)$ such that $q(r)=q(s)=0$ (such a basis always exists since the Arf invariant of $q$ is zero).

Propostrion 4.3. The isomorphism Aut $H_{1}\left(T^{2} ; \mathbb{Z}\right) \rightarrow \mathrm{OL}(2, \mathbb{Z})$, which consists in taking the matrix with respect to $\{r, s\}$, sends $H$ to the set of matrices $\left[\begin{array}{ll}\alpha & \beta \\ \gamma & \delta\end{array}\right]$ with $\alpha+\beta+\gamma+\delta$ even.

Remark 4.1. Notice that $\alpha+\beta+\gamma+\delta=$ even is not a condition invariant by change of basis.

Proof. Since $q(r+s)=r \cdot s=1$, the image of $H$ in $\mathrm{OL}(2, \mathbb{Z})$ is composed by the matrices $\left[\begin{array}{ll}\alpha & \beta \\ \gamma & \delta\end{array}\right]$ such that its reduction $\bmod 2$ stabilizes the vector of coordinates $(1,1)$. This is equivalent to the conditions $\alpha+\gamma \equiv \beta+\delta=1$ $(\bmod 2)$. In the presence of $\operatorname{det}\left[\begin{array}{ll}\alpha & \beta \\ \gamma & \delta\end{array}\right]= \pm 1$, this is equivalent to the condition $\alpha+\beta+\gamma+\delta=0(\bmod 2)$.

DEFINITION 4.4. $\left[\begin{array}{ll}\alpha & \beta \\ \gamma & \delta\end{array}\right]$ is even if $\alpha+\beta+\gamma+\delta$ is even.
COROLlary 4.5. The set of even matrices of OL $(2, \mathbb{Z})$ (or $\operatorname{SL}(2, \mathbb{Z})$ ) is a subgroup of index 3 which is not normal. The set $\left[\begin{array}{ll}1 & 0 \\ 0 & 1\end{array}\right],\left[\begin{array}{ll}1 & 1 \\ 0 & 1\end{array}\right],\left[\begin{array}{ll}1 & 0 \\ 1 & 1\end{array}\right]$ is a system of coset representatives.

Remark 4.2. If we call a column or row of $\left[\begin{array}{ll}\alpha & \beta \\ \gamma & \delta\end{array}\right]$ even if the sum of its elements is even, then two matrices are in the same left class (resp. right class) with respect to $H$ if and only if their rows (resp.columns) have the same parity.

Let us denote by $E$ (resp. $E^{+}$) the subgroup of even matrices of $\mathrm{OL}(2, \mathbb{Z})$ (resp. SL $(2, \mathbb{Z})$ ). Clearly $E^{+}$is a normal subgroup of index 2 of $E$, and $\left[\begin{array}{ll}1 & 0 \\ 0 & 1\end{array}\right],\left[\begin{array}{rr}1 & 0 \\ 0 & -1\end{array}\right]$ is a system of coset representatives. Let us describe a set of generators for $E^{+}$.

PROPOSTrION 4.6. If $\left[\begin{array}{ll}\alpha & \beta \\ \gamma & \delta\end{array}\right] \in E^{+}$then

i) $\alpha / \gamma$ has an expansion in continuous fraction

$$
\alpha / \gamma=a_{1}+\frac{1}{a_{2}+\cdots+\frac{1}{a_{m}}}
$$

with $a_{1}, \ldots, a_{m}$ even.

ii) There is an even number $a_{m+1}$ such that

$\left[\begin{array}{ll}\alpha & \beta \\ \gamma & \delta\end{array}\right]=$

$\begin{cases} \pm\left[\begin{array}{cc}1 & a_{1} \\ 0 & 1\end{array}\right]\left[\begin{array}{cc}1 & 0 \\ a_{2} & 1\end{array}\right] \cdots\left[\begin{array}{cc}1 & 0 \\ a_{m} & 1\end{array}\right]\left[\begin{array}{cc}1 & a_{m+1} \\ 0 & 1\end{array}\right] & \text { if } m=\text { even } \\ \pm\left[\begin{array}{rr}0 & -1 \\ 1 & 0\end{array}\right]\left[\begin{array}{cc}1 & 0 \\ -a_{1} & 1\end{array}\right]\left[\begin{array}{cc}1 & -a_{2} \\ 0 & 1\end{array}\right] \cdots\left[\begin{array}{cc}1 & 0 \\ -a_{m} & 1\end{array}\right]\left[\begin{array}{cc}1 & -a_{m+1} \\ 0 & 1\end{array}\right] & \text { if } m=\text { odd }\end{cases}$

Proof. We have

$$
\begin{gathered}
\alpha=a_{1} \gamma+r_{1} \\
\gamma=a_{2} r_{1}+r_{2} \\
\cdot \\
\cdot \\
\cdot \\
r_{m-3}=a_{m-1} r_{m-2}+r_{m-1} \\
r_{m-2}=a_{m}
\end{gathered}
$$


where $a_{1}, \ldots, a_{m-1}$ are even. Then

$$
\begin{aligned}
{\left[\begin{array}{cc}
1 & 0 \\
-a_{m} & 1
\end{array}\right]\left[\begin{array}{cc}
1 & -a_{m-1} \\
0 & 1
\end{array}\right] } & \cdots\left[\begin{array}{cc}
1 & 0 \\
-a_{2} & 1
\end{array}\right]\left[\begin{array}{cc}
1 & -a_{1} \\
0 & 1
\end{array}\right]\left[\begin{array}{ll}
\alpha & \beta \\
\gamma & \delta
\end{array}\right] \\
= & {\left[\begin{array}{cc}
1 & 0 \\
-a_{m} & 1
\end{array}\right] \cdots\left[\begin{array}{cc}
1 & 0 \\
-a_{2} & 1
\end{array}\right]\left[\begin{array}{ll}
r_{1} & * \\
\gamma & *
\end{array}\right]=\cdots= \pm\left[\begin{array}{ll}
1 & a \\
0 & 1
\end{array}\right] }
\end{aligned}
$$

for $m=$ even or

$$
\left[\begin{array}{cc}
1 & -a_{m} \\
0 & 1
\end{array}\right]\left[\begin{array}{cc}
1 & 0 \\
-a_{m-1} & 1
\end{array}\right] \cdots\left[\begin{array}{cc}
1 & 0 \\
-a_{2} & 1
\end{array}\right]\left[\begin{array}{cc}
1 & -a_{1} \\
0 & 1
\end{array}\right]\left[\begin{array}{cc}
\alpha & \beta \\
\gamma & \delta
\end{array}\right]= \pm\left[\begin{array}{cc}
0 & -1 \\
1 & a
\end{array}\right]
$$

$m=$ odd.

Since $a_{1}, \ldots, a_{m-1}$ are even and $\left[\begin{array}{ll}\alpha & \beta \\ \gamma & \delta\end{array}\right]$ is even, Remark 4.2 implies that the rows of $\left[\begin{array}{cc}1 & 0 \\ -a_{m} & 1\end{array}\right],\left[\begin{array}{ll}1 & a \\ 0 & 1\end{array}\right]$, when $m=$ even, (or the rows of $\left[\begin{array}{cc}1 & -a_{m} \\ 0 & 1\end{array}\right],\left[\begin{array}{cc}0 & -1 \\ 1 & a\end{array}\right]$, when $m=$ odd) have the same parity. Hence $a$ and $a_{m}$ are even.

Corollary 4.6. The set

$$
\left\{\left[\begin{array}{cc}
1 & \pm 2 \\
0 & 1
\end{array}\right],\left[\begin{array}{cc}
1 & 0 \\
\pm 2 & 1
\end{array}\right],\left[\begin{array}{cc}
0 & -1 \\
1 & 0
\end{array}\right],\left[\begin{array}{cc}
-1 & \\
& -1
\end{array}\right]\right\}
$$

is a system of generators for $E^{+}$

\section{Extension of automorphisms of $\partial E^{4}$}

Let $T^{2} \times[-1,1]$ be a regular neighbourhood in $\partial E^{4}$ of a meridian torus $T^{2}$ of the twin $\left(E^{4}, R, S\right)$. We identify $T^{2}$ with $T^{2} \times\{0\}$. Let $d$ be a canonical curve on $T^{2}$. We denote by $d^{\prime}$ (resp. $d^{\prime \prime}$ ) the curve $d \times\{\varepsilon\} \subset$ $T^{2} \times[-1,1]$ (resp. $d \times\{-\varepsilon\}$ ) with the natural framing, where $0<\varepsilon<1$.

LEMMA 5.1. Let $r_{1}, s_{1}$, be simple closed curves on $T^{2}$ such that

$$
\begin{aligned}
& r_{1} \sim \alpha r+\beta s \\
& s_{1} \sim \gamma r+\delta s \quad\left(\text { in } T^{2}\right)
\end{aligned}
$$

where $A=\left[\begin{array}{ll}\alpha & \beta \\ \gamma & \delta\end{array}\right]$ has determinant \pm 1 . Then there is a handle presentation

$$
E^{4}=\partial E^{4} \times[0,1] \cup 2 H^{2} \cup H^{3} \cup H^{4}
$$

where $2 H^{2}$ represents two 2 -handles attached to a collar $\partial E^{4} \times[0,1]$ of $\partial E^{4}$ along $r_{1}^{\prime} \times\{1\}$ and $s_{1}^{\prime \prime} \times\{1\} ; H^{3}$ is a 3-handle and $H^{4}$ is a 4-handle. Moreover, if $A$ is even, the addition of the two 2-handles is made using the natural framings. If $A$ is odd, one of the 2-handles is attached with the framing \pm 1 .

Proof. Let $c, d, \tilde{c}$ be simple closed curves in $T^{2}$, such that $\tilde{c} \sim c \pm 2 d$ (in $T^{2}$ ). In Figure 5.1 it is shown how $\tilde{c}^{\prime}$ (with natural framing) is obtained from $c^{\prime}$ by performing two band-moves with $d^{\prime \prime}$ (the figure only shows the case $\tilde{c}+2 d$; the other case is analogous). The move which consists on replacing the pair $(c, d)$ by $(\tilde{c}, d)$ will be called $\mathscr{F}^{2}$.

If $\tilde{c} \sim c \pm d$ (in $T^{2}$ ), then $\tilde{c}^{\prime}$ (with no natural framing) is obtained from
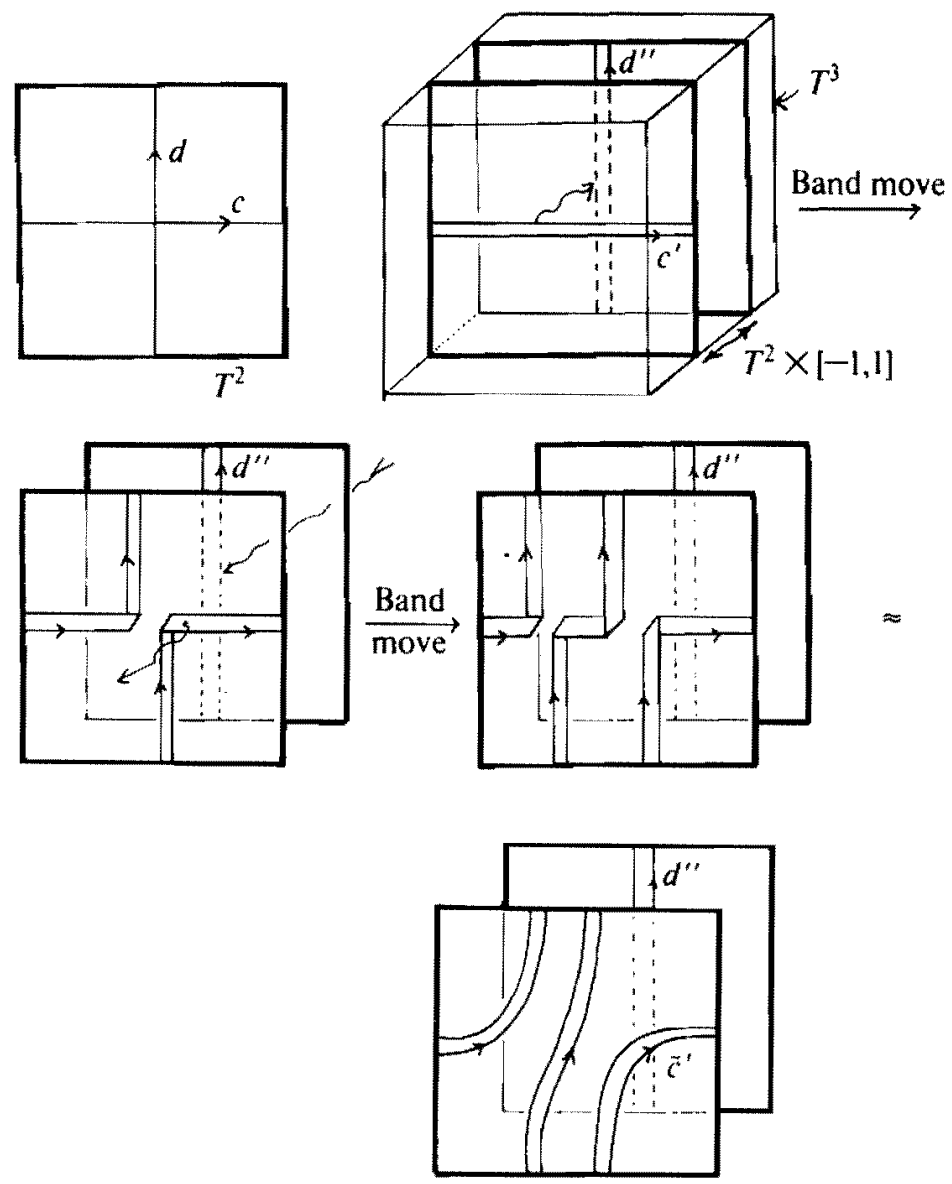

Fig. 5.1 

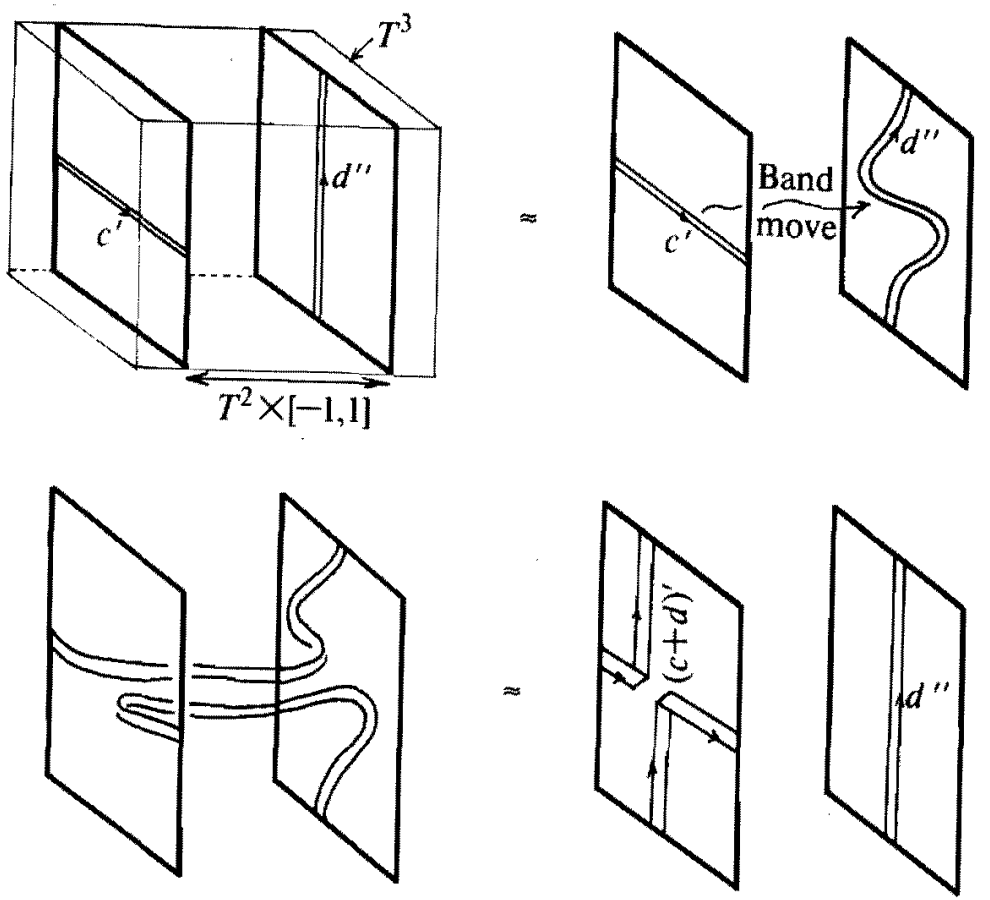

FIG. 5.2

$c^{\prime}$ by just one band-move with $d^{\prime \prime}$. We call $F$ the move which consists on replacing $(c, d)$ by $(\tilde{c}, d)$ (Figure 5.2 ).

Now, after eventually changing order and orientation of $(r, s)$, Corollary 4.6 implies that $A$ has one of the following forms

$$
B,\left[\begin{array}{ll}
1 & 1 \\
0 & 1
\end{array}\right] B, \quad\left[\begin{array}{ll}
1 & 0 \\
1 & 1
\end{array}\right] B
$$

where $B$ is a finite product of matrices of the form $\left[\begin{array}{cc}1 & \pm 2 \\ 0 & 1\end{array}\right]$ or $\left[\begin{array}{cc}1 & 0 \\ \pm 2 & 1\end{array}\right]$. a number of moves $\mathcal{F}^{2}$ actly when $A=B$. Thus, after applying to $\left(r^{\prime}, s^{\prime \prime}\right)$ $\left(r_{1}^{\prime}, s_{1}^{\prime \prime}\right)$ (with moves $\mathscr{F}^{2}$, and a unique move $F^{F}$ if not even, we get $\left(r_{1}^{\prime}, s_{1}^{\prime \prime}\right)$ (with the natural framings if and only if $A$ is even)

Now, in Section 3 we proved that $E^{4}=\partial E^{4} \times[0,1] \cup H_{1}^{2} \cup$ $H_{2}^{2} \cup H^{3} \cup H^{4}$, where the two 2-handles $H_{1}^{2}, H_{2}^{2}$ are attached along $r^{\prime}$ and $s^{\prime \prime}$ with the natural framings. Thinking of $\mathscr{F}^{2}$ and $\mathscr{F}$ as 2-handle slidings we see that $E^{4}=\partial E^{4} \times[0,1] \cup \hat{H}_{1}^{2} \cup \hat{H}_{2}^{2} \cup \hat{H}^{3} \cup \hat{H}^{4}$, where $\hat{H}_{1}^{2}, \hat{H}_{2}^{2}$ are
attached along $r_{1}^{\prime}, s_{1}^{\prime \prime}$ (using the even, then $\hat{H}_{1}^{2}$ (say) is at attached with the framing \pm 1 .
Let $W^{4}$ be a 4-manifold with non-empty boundary and let $E^{2}$ be a properly embedded dise in $W^{4}$. Pushing $E^{2}$ along a normal vector field without singularities, we obtain a framing for $\partial E^{2}$ in $\partial W^{4}$, which we call induced by $E^{2}$. We are interested in computing those framings for curves lying on $\partial E^{4}$.

COROLlaRY 5.2. Let $r_{1}$ be a simple closed curve on $T^{2}$ such that $r_{1} \sim \alpha r+\beta s$ (on $T^{2}$ ), and let $E^{2}$ be a properly embedded 2-disc in $E^{4}$ such that $\partial E^{2}=r_{1}$. Then, the framing of $r_{1}$ induced by $E^{2}$ differs from the canonical one in an even number of twists if and only if $\alpha+\beta$ is odd.

Proof. First, remark that two induced framings for $r_{1}$ differ in an even number of twists. To see this, write $E^{4}$ as $\overline{S^{4}-F^{2} \times D^{2}}$, where $F^{2}$ is a 2 -torus standardly embedded in $S^{4}$. There is a curve $\bar{r}_{1} \subset F^{2}$ and an annulus $A^{2} \subset F^{2} \times D^{2}$ which is normal to $F^{2} \cup T^{2}$ and such that $\partial A^{2}=$ $\bar{r}_{1} \cup r_{1}$. Take a vector field along $\bar{r}_{1}$ which is tangent to $F^{2}$ and extend it to $A^{2}$. This defines the natural framing on $r_{1}$. The obstruction $\bmod 2$ to extend this framing to $E^{2}$ is $q\left(\bar{r}_{1}\right)$, where $q$ is the quadratic form used in Section $4[R, 3]$. But that obstruction is equal to the difference, $\bmod 2$, between the canonical framing and the one induced by $E^{2}$.

Now assume $\alpha+\beta$ is odd. Then, there is an even matrix $\left[\begin{array}{ll}\alpha & \beta \\ \gamma & \delta\end{array}\right]=A$. By Lemma 5.1 there is a disc in $E^{4}$ inducing in $r_{1}$ the canonical framing.

If $\alpha+\beta$ is even, there is a matrix $A=\left[\begin{array}{ll}\alpha & \beta \\ \gamma & \delta\end{array}\right]$ with $\gamma+\delta$ odd. Hence $A=\left[\begin{array}{ll}1 & 1 \\ 0 & 1\end{array}\right] B, B$ even. Lemma 5.1 tells us that $r_{1}$ bounds a disc in $E^{4}$ inducing in $r_{1}$ the framing \pm 1 .

We apply these results to the problem of extending homeomorphisms $f: \partial E^{4} \rightarrow \partial E^{4}$ to $E^{4}$. Such an $f$ is isotopic to a linear homeomorphism $g$ of equation

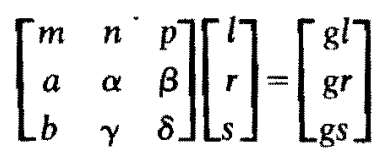

whose matrix $B$ has determinant +1 if and only if $g$ preserves the orientation of $\partial E^{4}$.

THEOREM 5.3. Let $\mathrm{g}: \partial E^{4} \rightarrow \partial E^{4}$ be a homeomorphism of matrix $B$. Then $g$ can be extended to a homeomorphism $G: E^{4} \rightarrow E^{4}$ if and only if $a=b=0$ and $A=\left[\begin{array}{ll}\alpha & \beta \\ \gamma & \delta\end{array}\right]$ is even.

Proof. First we see the sufficiency (see also remark 6.1). We have $\operatorname{det} A= \pm 1$. Then, by Lemma $5.1, E^{4}=\partial E^{4} \times[0,1] \cup H_{1}^{2} \cup H_{2}^{2} \cup S^{1} \times B^{3}$ 
and also $E^{4}=\partial E^{4} \times[0,1] \cup \hat{H}_{1}^{2} \cup \hat{H}_{2}^{2} \cup S^{1} \times B^{3}$, where $H_{i}^{2}$ are attached along $r^{\prime}, s^{\prime \prime}$ (with natural framings) and $\hat{H}_{i}^{2}$ are attached to $r_{1}^{\prime}, s_{1}^{\prime \prime}$ (with natural framings) where $\left[\begin{array}{l}r_{1} \\ s_{1}\end{array}\right]=A\left[\begin{array}{l}r \\ s\end{array}\right]$. The map $g$, being linear, preserves the natural framings, hence $g$ can be extended, first to $\partial E^{4} \times[0,1] \cup H_{1}^{2} \cup$ $H_{2}^{2} \rightarrow \partial E^{4} \times[0,1] \cup \hat{H}_{1}^{2} \cup \hat{H}_{2}^{2}$ and finally to $E^{4}$, since any homeomorphism of $S^{1} \times \partial B^{3}$ extends to $S^{1} \times B^{3}$

Conversely, if $g$ extends to $G$ we have the commutative diagram:

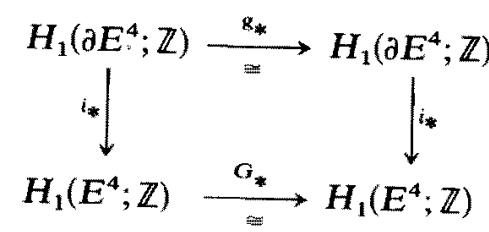

where $i_{*}$ is induced by inclusion. Hence $g_{*}\left(\right.$ kernel $\left.i_{*}\right)=\left(\right.$ kernel $\left.i_{*}\right)$. Since (kernel $i_{*}$ ) is generated by $r$ and $s$ we conclude that $a=b=0$.

Since $r$ and $s$ bound non singular dises $D^{2}, \tilde{D}^{2}$ properly embedded in $E^{4}$ inducing the natural framings in $r, s$ then $r_{1}, s_{1}$ bound discs $G\left(D^{2}\right)$ $G\left(D^{2}\right)$ inducing in $r_{1}, s_{1}$ the images by $g$ of the natural framings of $r, s$. Since $g$ is linear, these are the natural framings of $r_{1}, s_{1}$. Hence Corollary
5.2 implies that $A$ is even.

If $g: \partial E^{4} \rightarrow \partial E^{4}$ is a homeomorphism of matrix $B$ with $a=b=0$ and $A$ even, the extension $G$, provided by the above theorem, defines a new twin $\left(E^{4}, G(R), G(S)\right)$. The next problem is to derive this twin from $\left(E^{4}, R, S\right)$ by some sort of geometric transformation. Of course this will be obtained by realizing $\mathscr{F}^{2}$. This is done in the next Section.

We give now a partial converse of Proposition 4.1, which is a consequence of the above theorem.

THEOREM 5.4. Let $F^{2}$ be a torus embedded in $S^{3} \subset S^{4}$, and let $\varphi$ be an automorphism of $F^{2}$. Then $\varphi$ extends to an automorphism of $S^{4}$ if and only if $\varphi_{*}: H_{1}\left(F^{2} ; \mathbb{Z}\right) \rightarrow H_{1}\left(F^{2} ; \mathbb{Z}\right)$ belongs to $H$.

Proof. The "only if" part is proved in Proposition 4.1. To prove the "if" part note that, since the embedding $F^{2} \subset S^{4}$ is assumed to be standard, we have $S^{4}=D^{2} \times F^{2} \cup E^{4}$.

Now, let $r, s$ be curves on $T^{2}$ which are meridians of $E^{4}$. Then $q(\bar{r})=q(\bar{s})=0$ (proof of Corollary 5.2). Hence the matrix $A$ of $\varphi$ with respect to $r, s$ is even (Proposition 4.3).

Let us define $\psi=i d \times \varphi: D^{2} \times F^{2} \rightarrow D^{2} \times F^{2}$. Then $\partial \psi=\psi \mid \partial\left(D^{2} \times F^{2}\right)$ is isotopic to $g: T^{3} \rightarrow T^{3}$ with matrix $\left[\begin{array}{c|cc}1 & 0 & 0 \\ \hline 0 & A \\ 0 & A\end{array}\right]$ with respect to the basis $\left(\partial D^{2}, r, s\right)$. But $g$ extends to $E^{4}$ because $A$ is even (Theorem 5.3).
It remains to be investigated what happens when $F^{2}$ is not standardly embedded in $S^{4}$.

We finish the section with an easy consequence of Theorem 5.3.

Definition 5.5. Let $F^{2}$ be a 2-torus embedded in $S^{4}$ and let $N\left(F^{2}\right)$ be a regular neighbourhood of $F^{2}$ in $S^{4}$. We will say that the manifold $S^{4}-N\left(F^{2}\right) \cup_{i \varphi} N\left(F^{2}\right)$, where $\varphi: \partial N\left(F^{2}\right) \rightarrow \partial N\left(F^{2}\right)$ is an automorphism and $i$ is the natural identification between boundaries, is obtained by Dehn-surgery on $F^{2}$.

Notice that $N\left(F^{2}\right)=F^{2} \times D^{2}$ has a handle presentation made of one 0 -handle, two 1-handles and one 2-handle with belt sphere the curve $l=\{*\} \times \partial D^{2},\{*\} \in F^{2}$. Hence $\overline{S^{4}-N\left(F^{2}\right)} \cup_{i \varphi} N\left(F^{2}\right)$ depends only on the canonical curve $i \varphi(l)$ (with canonical framing). In fact, we attach a 2-handle to $\overline{S^{4}-N\left(F^{2}\right)}$ along the framed $i \varphi(l)$, and after that we glue $S^{1} \times B^{3} b S^{1} \times B^{3}$ arbitrarily to get $\bar{S}^{4}-N\left(F^{2}\right) \cup_{i \varphi} N\left(F^{2}\right)$.

Assume $F^{2}$ is standardly embedded in $S^{4}$. Then we can assume $\overline{s^{4}-N\left(F^{2}\right)}$ is $E^{4}$; let $r, s$ be meridians of $E^{4}$. Here $i \varphi(l) \sim m l+n r+p s$ (on $\left.\partial E^{4}\right)$ and we call $(m, n, p)$ the surgery data. The fundamental group of the manifold $W^{4}$ obtained by Dehn-surgery in $F^{2}$ with surgery data $(m, n, p), m \geqslant 0$, is $\mathbb{Z} / m \mathbb{Z}$. Hence, this manifold is simply connected when $m=1$ and, in this case, $H_{2}\left(W^{4}\right)=0$ by Mayer-Vietoris. Hence $W^{4} \simeq S^{4}$ if and only if $m=1$.

COROLLARY 5.6. Let $F^{2}$ be a 2-torus standardly embedded in $S^{3} \subset S^{4}$. Then, any homotopy 4-sphere obtained by Dehn-surgery on $F^{2}$ is $S^{4}$.

Proof. Let $W^{4}$ be the result of Dehn-surgery in $F^{2}$ with data $(1, n, p)$. Then $W^{4}=E^{4} \cup_{j \psi} F^{2} \times D^{2}$, where $j$ is the canonical identification and $\psi$ is an automorphism of $\partial E^{4}$ of equation

$$
\left[\begin{array}{l}
\psi l \\
\psi r \\
\psi s
\end{array}\right]=\left[\begin{array}{ccc}
1 & -n & -p \\
0 & 1 & 0 \\
0 & 0 & 1
\end{array}\right]\left[\begin{array}{l}
l \\
r \\
s
\end{array}\right]
$$

In fact, $\psi(l+n r+p s)=l$. But, $\psi$ extends to an automorphism of $E^{4}$, hence $W^{4} \cong S^{4}$.

\section{Two-spinning $R$ around $S$}

Let $N(R)$ be a regular neighbourhood of $R$ in $E^{4}$. Then $\partial N(R)=$ $S^{1} \times S^{2}$ separates $E^{4}$ in two regions. One of them is $N(R)=D^{2} \times S^{2}$, the other is $S^{1} \times V^{3}$, where $V^{3}$ is a punctured solid torus. We can identify $V^{3}$ with $B^{3}$ minus the interior of a regular neighbourhood of a simple unknotted curve which is invariant by rotations around the poles.

The boundary of $S^{1} \times V^{3}$ is the disjoint union of $S^{1} \times S^{2}$ and $T^{3}=\partial E^{4}$. 
The automorphism $\tau$ extends to an automorphism $\vec{\tau}$ of $S^{1} \times V^{3}$ which induces in $\partial E^{4}$ the automorphism of matrix $\left[\begin{array}{lll}1 & 0 & 0 \\ 0 & 1 & 1 \\ 0 & 0 & 1\end{array}\right]$.

It is known [GL] that $\tau^{2 k}$ is isotopic to the identity. Let $F: S^{1} \times S^{2} \times$ $[0,1] \rightarrow S^{1} \times S^{2} \times[0,1]$ be the isotopy. Then, identifying $S^{1} \times S^{2} \times[0,1]$ with a collar of $\partial N\left(R^{2}\right), F$ extends to an automorphism $G$ of $N(R)$. Hence the automorphism $\psi$ of $E^{4}$ defined by $\psi|N(R)=G, \psi| S^{1} \times V^{3}=$ $\bar{\tau}^{2 k}$ induces in $\partial E^{4}$ an automorphism of matrix $\left[\begin{array}{ccc}1 & 0 & 0 \\ 0 & 1 & 2 k \\ 0 & 0 & 1\end{array}\right]$. We will say that $\psi$ is the $2 k$-spinning of $S$ around $R$. We define analogously the $2 k$-spinning of $R$ around $S$.

Let $g$ be an automorphism of $\partial E^{4}$ with matrix $B=\left[\begin{array}{ccc}m & n & p \\ 0 & \alpha & \beta \\ 0 & \gamma & \delta\end{array}\right]$, where $A=\left[\begin{array}{ll}\alpha & \beta \\ \gamma & \delta\end{array}\right]$ is even, and let $G: E^{4} \rightarrow E^{4}$ be the extension of $g$ given by Theorem 5.3. Let $\tilde{R}$ and $\tilde{S}$ be the images of $R, S$ by $G$.

Corollary 6.1. $(\tilde{R}, \tilde{S})$ is obtained from $(R, S)$ by a finite sequence of $2 k$-spinnings.

Proof. Since $\tilde{R}, \tilde{S}$ are determined by their meridians (see the end of Section 3 ), instead of using $g$ we can use an automorphism with matrix $\left[\begin{array}{l|ll}1 & 0 & 0 \\ \hline 0 & A \\ 0 & A\end{array}\right]$ which is a product of a finite number of matrices of type $\left[\begin{array}{rrr}1 & 0 & 0 \\ 0 & 1 & \pm 2 \\ 0 & 0 & 1\end{array}\right]$ or $\left[\begin{array}{rrr}1 & 0 & 0 \\ 0 & 1 & 0 \\ 0 & \pm 2 & 1\end{array}\right]$ (see the proof of Lemma 5.2). From here the corollary follows.

Remark 6.1. Notice that above we have reproved the sufficiency part of Theorem 5.3 , in a constructive way.

We want now to visualize the effect of the 2-spinning of $S$ around $R$. The intersection of $S$ with the collar $S^{1} \times S^{2} \times[0,1]$ of $N(R)$ is $S^{1} \times S^{0} \times$ $[0,1]$, where $S^{0}$ are the north and south poles of $S$. On the other hand, the automorphism $\bar{\tau}^{2}$ of $S^{1} \times V^{3}$ fixes $\left(S^{1} \times V^{3}\right) \cap S$. Hence the 2-spinning $\psi$ fixes $R$ and $\psi(S)=\left[\overline{E^{4}-S^{1} \times S^{2} \times[0,1]} \cap S\right] \cup F\left(S^{1} \times S^{0} \times[0,1]\right)$. Thus, we only have to visualize the effect of $F$ in the pair $\left(S^{1} \times S^{2} \times[0,1], S^{1} \times\right.$ $\left.S^{0} \times[0,1]\right)$ and we have done this in Section 1 . Thus, to visualize the

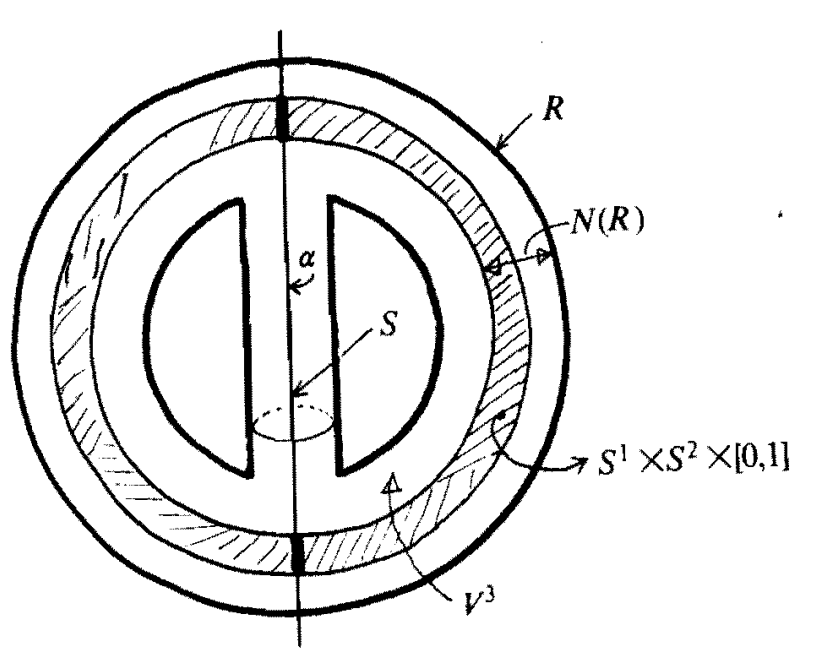

FIG. 6.1

"total" effect of the 2-spinning $\psi$ we use a "movie" picture of the twin. "The punctured solid torus of Figure 6.1 around This is done by spinning the spinning, the component $S$ of the twin is created the $R$-boundary. In the spinning, the componed region of Figure 6.1 we by the arc $\alpha$. Now if in the spinning of the shaded region of Figure 6.1 we
introduce Figure 1.2 we obtain a picture of the 2 -spinning of $S$ around $R$.

\section{Twins in 4-manifolds}

Let $M$ be a closed 4-manifold. By a twin in $M$ we will mean a subset $R \cup S$ of $M$ which consists of two 2-spheres meeting transversally in two $R \cup S$ of $M$ which consists of two number zero (automatic if $\mathrm{H}_{2}(M ; \mathbb{Z})=0$ ). Then $E^{4}$ can be identified with a regular neighbourhood of the twin in $M$. We will use two notations for a twin in $M,(M, E, R, S)$ or simply $R \cup S$. The two coordinate planes $R=\mathbb{R}^{2} \times\{0\}$ and $S=\{0\} \times \mathbb{R}^{2}$ in $S^{4}=$ $\mathbb{R}^{4}+\{\infty\}$ define the trivial twin in $S^{4}$.

EXAMPLES. a) Let $S^{4}=B^{3} \times S^{1} / J$, where the identification $J$ collapses $\{x\} \times S^{1}$ to a point for every $x$ in $\partial B^{3}$. Let $A$ be a knotted spanning are in $B^{3}$. Then $A \times S^{1} / J$ is a $2-k n o t S$ in $S^{4}$. Let $R$ be $\partial B^{3}$. Then $R \cup S$ is called the spun twin of $A$, which is non trivial unless $A$ be unknotted.

b) If in example a) the arc $A$ spins $n$ times around itself while it spins b) If $R$ we get the $n$-twist spun twin of $A$. The 1-twist spun twin of $A$ is a twin $R \cup S$ where both $R$ and $S$ are trivial knots. However the twin is not trivial. 
c) Let $R \cup S$ be the spun twin of $A$. Let $\tilde{S}$ be the result of $2 k$-spinning $S$ around $R$ (Section 6). Then, we have seen in Section 1 that $R \cup \tilde{S}$ is the $2 k$-twist spun twin of $A$.

\section{Surgery in a twin in $S^{4}$}

Let $R \cup S$ be a twin in $S^{4}$. Let $r, s$ be a system of meridians in $\partial E^{4}$. Let $X^{4}$ be the exterior $S^{4}$-int $E^{4}$ of the twin. Alexander duality gives $\tilde{H}_{i}\left(X^{4} ; \mathbb{Z}\right) \cong \tilde{H}_{3-i}\left(E^{4} ; \mathbb{Z}\right)$. Thus $X^{4}$ is homologically $D^{2} \times S^{1} \times S^{1}$ and $r, s$ represent generators of $H_{1}\left(X^{4} ; \mathbb{Z}\right)$. Then, up to isotopy, there is a unique canonical simple closed curve $l$ in $\partial E^{4}$ which is nulhomologous in $X^{4}$, such that $l, r, s$ represent a basis of $H_{1}\left(\partial E^{4} ; \mathbb{Z}\right)$. Assume we have identified $\partial E^{4}$ with $T^{3}$ so as to have $l=S^{1} \times\{*\} \times\{*\}, r=\{*\} \times S^{1} \times\{*\}$, We $\times\{*\} \times S^{1}$. We call l longitude of the twin.

We will use the description of a twin made at the end of Section 3 . Namely we think of $\left(S^{4}, E^{4}, R, S\right)$ as $X^{4}$ with the identifications in the boundary explained in that section. We will use the notation $X^{4} /(r, s)$ to mean the twin $\left(S^{4}, E^{4}, R, S\right)$. We will use also $X^{4} /(-, s)$ or $X^{4} /(r,-)$ to mean the exterior of $R$ or $S$, respectively.

Let $r_{1}=(a, \alpha, \beta), s_{1}=(b, \gamma, \delta)$ be canonical curves on $T^{3}=\partial X^{4}$ which are part of a basis (i.e. there are integers $m, n, p$ such that $\left|\begin{array}{lll}m & n & p \\ a & \alpha & \beta \\ b & \gamma & \delta\end{array}\right|=$ 1) and assume they are oriented so as to have $\left|\begin{array}{ll}\alpha & \beta \\ \gamma & \delta\end{array}\right| \geqslant 0$. Then $X^{4} /\left(r_{1}, s_{1}\right)$ is homeomorphic to $\left(X^{4} \cup_{i \varphi} E^{4}, E^{4}, R, S\right)$, where $\varphi$ is given by $\left[\begin{array}{lll}m & n & p \\ a & \alpha & \beta \\ b & \gamma & \delta\end{array}\right]$ and $i: \partial X^{4} \rightarrow \partial E^{4}$ is the natural identification. Thus $X^{4} /\left(r_{1}, s_{1}\right)$ is a knotted twin in the space $X^{4} \cup \cup_{i \varphi} E^{4}$ which we say is obtained by $\left(r_{1}, s_{1}\right)$-surgery on $E^{4}$.

In the case $a=b=0$ we say the $\left(r_{1}, s_{1}\right)$-surgery is special, otherwise we will call it general.

We, first, study the case of special surgery. Then $\varphi$ is given by $\left[\begin{array}{lll}1 & 0 & 0 \\ 0 & \alpha & \beta \\ 0 & \gamma & \delta\end{array}\right]\left[\begin{array}{l}l \\ r \\ s\end{array}\right]=\left[\begin{array}{l}l_{1} \\ r_{1} \\ s_{1}\end{array}\right]$

THEOREM 8.1. If $\left(r_{1}, s_{1}\right)$ satisfies $\left[\begin{array}{l}r_{1} \\ s_{1}\end{array}\right] \sim\left[\begin{array}{ll}\alpha & \beta \\ \gamma & \delta\end{array}\right]\left[\begin{array}{l}r \\ s\end{array}\right]$ in $\partial X^{4}$ and $A=$ $\left[\begin{array}{ll}\alpha & \beta \\ \gamma & \delta\end{array}\right]$ is even, then $X^{4} /\left(r_{1}, s_{1}\right)$ is a knotted twin $\left(S^{4}, \tilde{E}^{4}, \tilde{R}, \tilde{S}\right)$ in $S^{4}$.
Moreover $\left(S^{4}, \tilde{E}^{4}\right)$ can be identified with $\left(S^{4}, E^{4}\right)$ in such a way that $\tilde{R}, \tilde{S}$ is obtained by a finite sequence of $2 k$-spinnings in $\left(E^{4}, R, S\right)$.

Definition 8.2. When $A=\left[\begin{array}{cc}1 & 2 k \\ 0 & 1\end{array}\right], \tilde{R}=R$ and we say that $\tilde{S}$ is the $2 k$-twist spun knot of $S$ with respect to $R$. This generalizes partially the definition of Zeemann [Z] (compare example c) in Section 7).

The situation changes if $A$ is not even. From Section 4 we have $A=B\left[\begin{array}{ll}1 & 1 \\ 0 & 1\end{array}\right]$ or $B\left[\begin{array}{ll}1 & 0 \\ 1 & 1\end{array}\right]$ where $B$ is even. Let us take the following automorphisms of $\partial X^{4}: \rho=\left[\begin{array}{lll}1 & 0 & 0 \\ 0 & 1 & 1 \\ 0 & 0 & 1\end{array}\right], \sigma=\left[\begin{array}{lll}1 & 0 & 0 \\ 0 & 1 & 0 \\ 0 & 1 & 1\end{array}\right]$. Then we have the knotted twins $X^{4} /(\rho r, \rho s)$ and $X^{4} /(\sigma r, \sigma s)$.

THEOREM 8.3. Let $\left(r_{1}, s_{1}\right)$ with $\left[\begin{array}{l}r_{1} \\ s_{1}\end{array}\right] \sim\left[\begin{array}{ll}\alpha & \beta \\ \gamma & \delta\end{array}\right]\left[\begin{array}{l}r \\ s\end{array}\right]$ in $\partial X^{4}$ and $\left[\begin{array}{ll}\alpha & \beta \\ \gamma & \delta\end{array}\right]=$ $B\left[\begin{array}{ll}1 & 1 \\ 0 & 1\end{array}\right]$ (resp. $\left.B\left[\begin{array}{ll}1 & 0 \\ 1 & 1\end{array}\right]\right)$ where $B$ is even. Then the twin $X^{4} /\left(r_{1}, s_{1}\right)$ can be identified with the result of a finite sequence of $2 k$-spinnings in $X^{4} /(\rho r, \rho s)$ (resp. $\left.X^{4} /(\sigma r, \sigma s)\right)$. Moreover $X^{4} /\left(r_{1}, s_{1}\right)$ is a twin in $S^{4}$ if and only if $X^{4} /(\rho r, \rho s)\left(\right.$ resp. $\left.X^{4} /(\sigma r, \sigma s)\right)$ is.

Proof. Let $\left[\begin{array}{ll}\alpha & \beta \\ \gamma & \delta\end{array}\right]=B\left[\begin{array}{ll}1 & 1 \\ 0 & 1\end{array}\right]$ and let $\psi=\left[\begin{array}{l|ll}1 & 0 & 0 \\ \hline 0 & B \\ 0 & B\end{array}\right]$. Let $C^{4}=$ $\partial E^{4} \times[0,1]$ a collar of $\partial E^{4}\left(=\partial E^{4} \times\{0\}\right)$ in $E^{4}$. Let $\tilde{E}^{4}=\overline{E^{4}-C^{4}}$. Then $X^{4} /\left(r_{1}, s_{1}\right)$ is $\left(X^{4} \cup_{i \text { iup }} C^{4} \cup \tilde{E}^{4}, \tilde{E}^{4}, R, S\right)$. This is homeomorphic to $\left(X^{4} \cup_{i \rho} C^{4} \cup_{\psi} \tilde{E}^{4}, \tilde{E}^{4}, R, S\right)$, defining the homeomorphism to be the identity in $X^{4}$ and $\tilde{E}^{4}$, and equal to $\psi^{-1} \times i d$ in $C^{4}$. The last twin is homeomorphic to $\left(X^{4} \cup \cup_{i \mathrm{\rho}} C^{4} \cup \tilde{E}^{4}, \tilde{E}^{4}, \Psi^{-1} R, \Psi^{-1} S\right)$ where $\Psi$ is an extension of $\psi$ to $\tilde{E}^{4}$, which certainly exists since $B$ is even. Hence $\Psi^{-1} R, \Psi^{-1} S$ come from $R, S$ by a finite sequence of $2 k$-spinnings in $\left(X^{4} \cup_{i \rho} E^{4}, E^{4}, R, S\right)=X^{4} /(\rho r, \rho s)$. Since $B^{-1}$ is also even, the converse follows.

In the next theorem we use the notation of Gordon [G], i.e. we call $R^{*}$ the result of Gluck-surgery on $R \subset S^{4}$ (see [GL]). $S^{*}$.

THEOREM 8.4. $X^{4} /(\rho r, \rho s)$ is a twin in $R^{*}$ and $X^{4} /(\sigma r, \sigma s)$ is a twin in

Proof. $\left(X^{4} \cup_{i \rho} E^{4}\right)=\left(X^{4} \cup_{i \rho} S^{1} \times V^{3} \cup N(R)\right) \cong\left(X^{4} \cup_{i} S^{1} \times V^{3} \cup_{T} N(R)\right)$, defining the homeomorphism as the identity in $X^{4}$ and $N(R)$, and $\bar{\tau}$ in $S^{1} \times V^{3}$. Recall that $\tilde{\tau} \mid \partial E^{4}=\rho$ (Section 6). 
If $R \cup S$ is the twin of example a), Section 7 , then $X^{4} /(\rho r, \rho s)$ is the 1 -twist spun twin of example b). This remark justifies the following definition.

Definition 8.5. When $A=\left[\begin{array}{cc}1 & 2 n+1 \\ 0 & 1\end{array}\right]$ we say that $\tilde{S}$ is the $(2 n+1)$ twist spun knot of $S$ with respect to $R$. Note that $\bar{S}$ is a $2-k n o t$ in $R^{*}$. This completes the generalization of Zeeman's definition $[\mathbf{Z}]$.

COROLlary 8.6. If in the twin $\left(S^{4}, E^{4}, R, S\right), R, S$ are both trivia $2-k n o t s$ in $S^{4}$, then the twins obtained by special surgery are twins in $S^{4}$ These twins fall into three families, namely those obtained by $2 k$-spinnings on $\left(S^{4}, E^{4}, R, S\right), X^{4} /(\rho r, \rho s)$ or $X^{4} /(\sigma r, \sigma s)$.

Remark 8.1. If $K$ is a 2 -knot of any of the twins of the three families, then $K^{*} \cong S^{4}$. This generalizes [G; Theorem 3.1].

Remark 8.2. A twin is called interchangeable if there is a homeomorphism sending $\left(S^{4}, E^{4}, R, S\right)$ to $\left(S^{4}, E^{4}, S, R\right)$. In an interchangeable twin, the twins obtained by special surgery fall in only two families. An example of interchangeable twin is provided by the 1-twist spun twin of a spanning arc on $B^{3}$ (example b) of Section 7). This is proved in [GK]. We now give a different proof.Let $K$ be a knot in $S^{3}$ and let $W$ be the exterior of $K$. Let $r$ be a meridian of $K$. Let $X=W \times S^{1}$ and let $s \subset \partial X$ be $\{x\} \times S^{1}$. Let $s_{1}$ be a canonical curve in $\partial X$ homologous to $s+r$. In $X /\left(r, s_{1}\right)$ the $2-\operatorname{knot} R_{1}$ of meridian $r$ is the 1 -twist spun knot of $K$. The automorphism of $X$ defined by $(w, z) \mapsto(w, f w-z)$ sends $s_{1}$ to $r$ and $r$ to $s_{1}$, where $f: W \rightarrow S^{1}$ is a map which restricted to $\partial W$ is projection onto $r$. Hence there is an automorphism of $X /\left(r, s_{1}\right)$ which permutes $R_{1}$ and $S$.

Now we study the case of general surgery. We have to understand $X^{4} /\left(r_{1}, s_{1}\right)$, where

$$
\begin{aligned}
& r_{1}=(a, \alpha, \beta) \\
& s_{1}=(b, \gamma, \delta)
\end{aligned}
$$

are canonical curves on $T^{3}=\partial X^{4}$ which are part of a basis.

PropostTION 8.7. $X^{4} /\left(r_{1}, s_{1}\right)$ is a twin in a homology 4-sphere if and only if $\alpha \delta-\beta \gamma= \pm 1$.

Proof. Since $r, s$ is a basis of $H_{1}\left(X^{4} ; \mathbb{Z}\right)$, the group $H_{1}\left(X^{4} /\left(r_{1}, s_{1}\right) ; \mathbb{Z}\right)$ has generators $r, s$ and relations $\alpha r+\beta s=0, \gamma r+\delta s=0$. Hence it is trivial if and only if $\alpha \delta-\beta \gamma= \pm 1$. In this case, an argument involving MayerVietoris, Poincaré duality and the universal coefficient theorem, shows that also $H_{2}\left(X^{4} /\left(r_{1}, s_{1}\right) ; \mathbb{Z}\right)$ is trivial.
Thus we restrict our attention to general surgery $X^{4} U_{i \varphi} E^{4}$, where $\varphi$ is given by

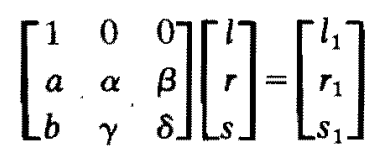

and $\left|\begin{array}{cc}\alpha & \beta \\ \gamma & \delta\end{array}\right|=1$

We study first the general surgery corresponding to the matrices $\omega(a, b)=\left[\begin{array}{lll}1 & 0 & 0 \\ a & 1 & 0 \\ b & 0 & 1\end{array}\right]$. To do this, take a collar $T^{3} \times[0,1]$ of $T^{3} \times\{0\}=$ $\partial E^{4}$, and consider $V_{r}=S^{1} \times S^{1} \times\{*\} \times\left[0, \varepsilon_{1}\right]$ and $V_{s}=S^{1} \times\left\{*^{\prime}\right\} \times S^{1} \times\left[0, \varepsilon_{2}\right]$ with $\varepsilon_{1} \neq \varepsilon_{2}$. Then $V_{r} \cap V_{s}$ is an annulus, and $\partial V_{r}-\partial E^{4}=T_{r}, \partial V_{s}-\partial E^{4}=T_{s}$ are disjoint tori (Figure 8.1).

Remark 8.3. $T_{r}$ is isotopic to the torus obtained by replacing a regular neighbourhood $N(S \cap R)$ of $S \cap R$ in $S$ with a tube whose core is a suitable simple closed curve on $R$, connecting the points of $S \cap R$ (see Figure 8.1).

We take the following "coordinates" on $\partial N\left(T_{r}\right)$ : the meridian of $N(T)$ and two oriented curves, which are parallel (not antiparallel) to $l, r$; moreover, we orient the meridian of $N\left(T_{r}\right)$ to induce with $l, r$ the orientation of $\partial N(T)$ (as boundary of $N(T)$ ). We define coordinates for $\partial N\left(T_{\mathrm{s}}\right)$ in the same way.

Propostrion 8.8. $X^{4} /(\omega(a, b) r, \omega(a, b) s)$ is the twin obtained by performing Dehn-surgery on $T_{s}$ with surgery data $(1, a, 0)$ and Dehn-surgery on $T_{r}$ with surgery data $(1, b, 0)$.

Proof. We adapt the trick of Lickorish [LK]. Assume $\varepsilon_{1}>\varepsilon_{2}$. We cut $E^{4}$-int $N(T)$ along $V_{r}$ and we define an automorphism on a collar $V_{r} \times[0,1]$ of one "end" as follows: for each $t \in\left[0, \varepsilon_{1}\right]$, that automorphism is an isotopy from the identity on $S^{1} \times S^{1} \times\{*\} \times\{t\} \times\{1\}$ to a rotation of $b$ times $360^{\circ}$ in which the orbit of a point is $S^{1} \times\{*\} \times\{*\} \times\{t\} \times\{0\}$. Then we paste the two ends back together. The resulting automorphism on $\partial E^{4}$ is given by the matrix $\left[\begin{array}{lll}1 & 0 & 0 \\ 0 & 1 & 0 \\ b & 0 & 1\end{array}\right]$, and the meridian of $N\left(T_{r}\right)$ goes to the canonical curve $(1, b, 0)$. Finally, we repeat this by using $T_{s}$ instead of $T_{\text {r }}$ and $a$ instead of $b$.

DEFINITION 8.9. We say that the twin $X^{4} /(\omega(a, b) r, \omega(a, b) s)$ is the $(a, b)$-rolling of $(S, R)$ around $(R, S)$. 


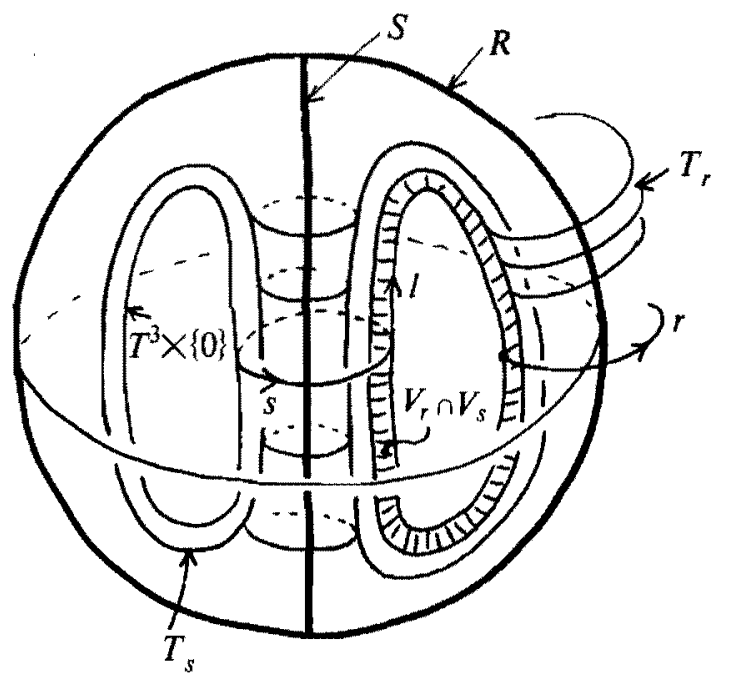

FIG. 8.1a

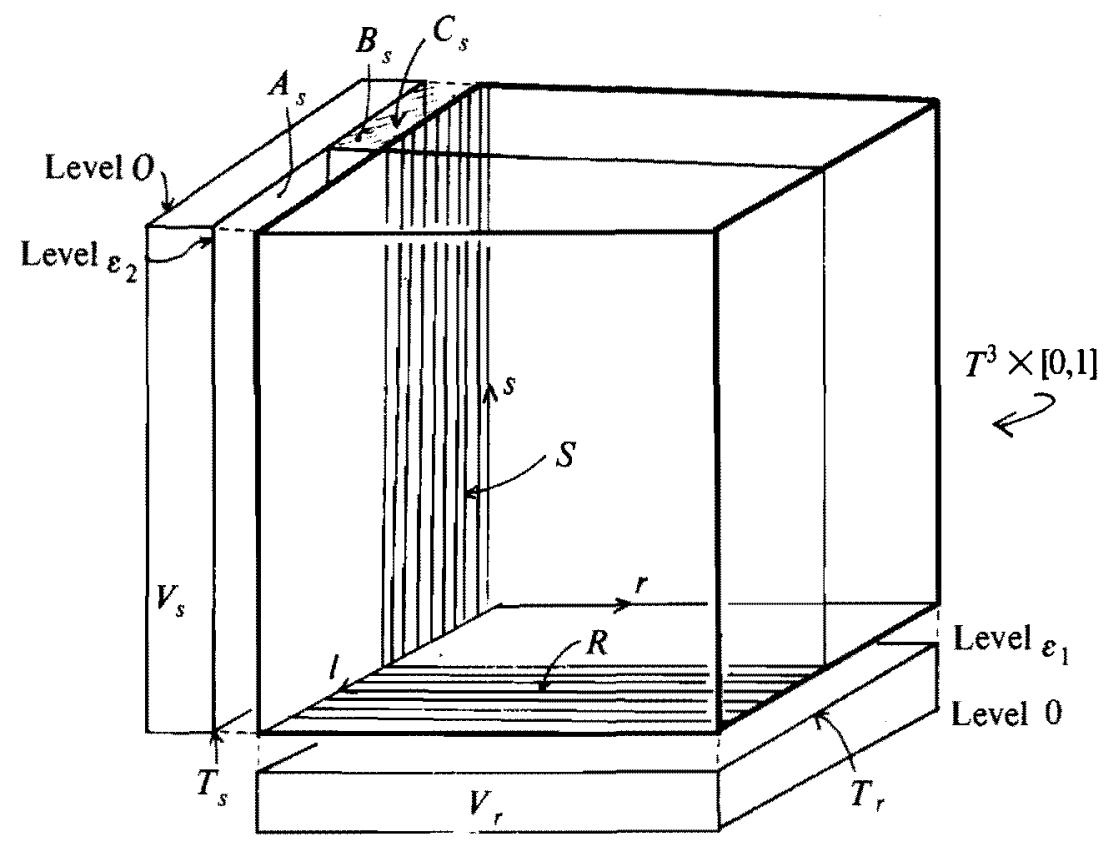

Fig. $8.1 \mathrm{~b}$
To justify this definition let us consider the $(a, 0)$-rolling of $(S, R)$ around $(R, S)$. This is the result of Dehn-surgery on $T_{s}$ with surgery data $(1, a, 0)$. We now describe this Dehn-surgery in a different way. As at the beginning of Section 6 we write $E^{4}=N\left(R^{2}\right) \cup S^{1} \times V^{3}$. Let $T_{s} \times[0,1]$ be beginning of Section 6 we write $E^{4}=N\left(R^{2}\right) S^{3}$. We define an automorphism $\lambda$ of $x \times[0,1]$ as follows: $(x, y, t) \mapsto(t a+x, y, t)$, where $(x, y)$ are coordiof $T_{s} \times[0,1]$ as respect to $l$ and $r$, and $t \in[0,1]$. Cut $E^{4}$ along $T_{s} \times[0,1]$ and paste the two ends back together using $\lambda$. The result is Dehn-surgery on $T_{s}$ with surgery instructions $(1, a, 0)$ (Figure 8.2).
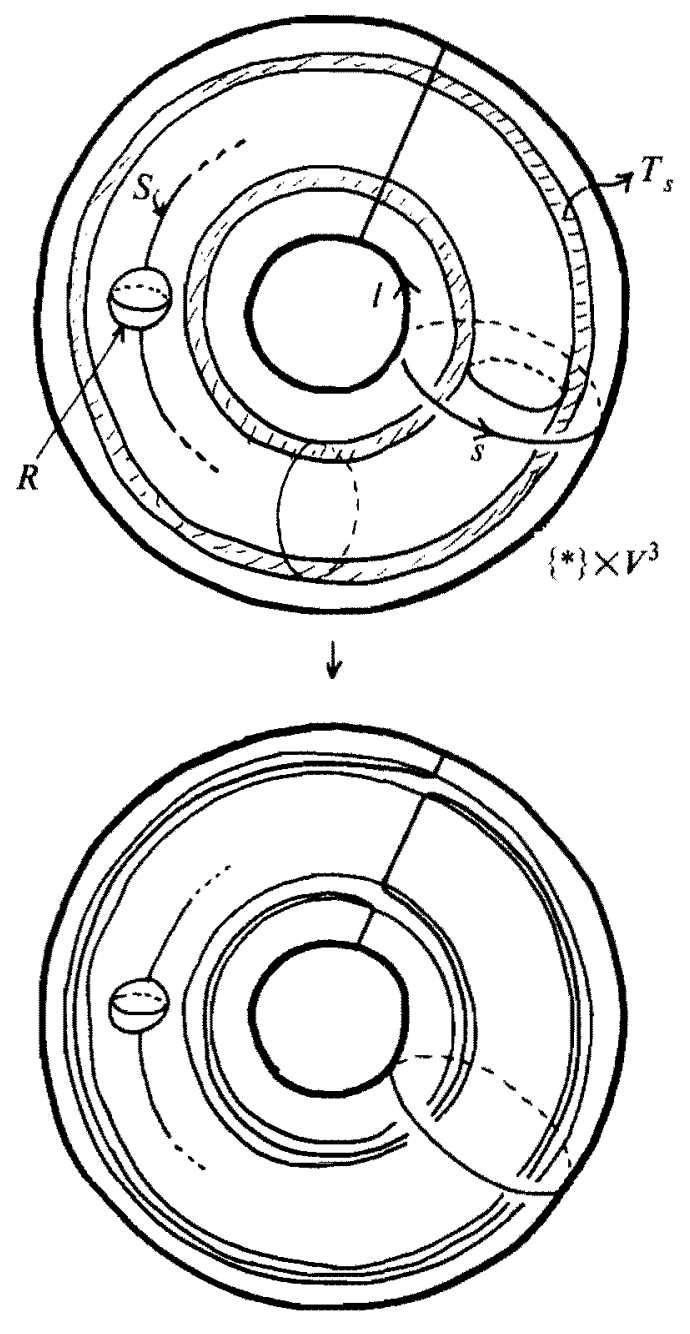

FIG. 8.2 
When the twin $R \cup S$ is example a), Section 7, the above construction modifies the knot $S$ giving "the $a$-rolling of $S^{2}$ " in the language of Litherland [L]. The knot $R$ does not change.

COROLlaRY 8.10. The general surgery corresponding to the matrix $\left[\begin{array}{lll}1 & 0 & 0 \\ a & \alpha & \beta \\ b & \gamma & \delta\end{array}\right]$, is the result of a number of spinnings, determined by $\left[\begin{array}{ll}\alpha & \beta \\ \gamma & \delta\end{array}\right]$ and one $(a, b)$-rolling.

Proof. This is a consequence of the

$$
\left[\begin{array}{lll}
1 & 0 & 0 \\
a & \alpha & \beta \\
b & \gamma & \delta
\end{array}\right]=\left[\begin{array}{lll}
1 & 0 & 0 \\
a & 1 & 0 \\
b & 0 & 1
\end{array}\right]\left[\begin{array}{lll}
1 & 0 & 0 \\
0 & \alpha & \beta \\
0 & \gamma & \delta
\end{array}\right]
$$

General surgery with matrix $A=\left[\begin{array}{ccc}1 & 0 & 0 \\ a & \alpha & \beta \\ b & \gamma & \delta\end{array}\right],\left|\begin{array}{ll}\alpha & \beta \\ \gamma & \delta\end{array}\right|=1$ gives a twin in a homology 4-sphere. We now describe, some particular cases for which this homology 4 -sphere is actually $S^{4}$. The general case is still an open problem.

LeMMA 8.11. Let $A=\left[\begin{array}{lll}1 & 0 & 0 \\ a & \alpha & \beta \\ b & \gamma & \delta\end{array}\right]$ with $\left|\begin{array}{ll}\alpha & \beta \\ \gamma & \delta\end{array}\right|=1$, and $d=(a, b)$. Then $A=\left[\begin{array}{c|cc}1 & 0 & 0 \\ \hline 0 & P \\ 0 & P\end{array}\right] B$, where $P$ is even, $\operatorname{det} P=1$ and $B$ is $\left[\begin{array}{c|cc}1 & 0 & 0 \\ \hline d & Q \\ 0 & Q\end{array}\right]$ if $a / d+b / d$ is odd or $\left[\begin{array}{l|ll}1 & 0 & 0 \\ \hline d & Q \\ d & Q\end{array}\right]$ if $a / d+b / d$ is even.

This lemma reduces our research to general surgery $\left[\begin{array}{ccc}1 & 0 & 0 \\ a & \alpha & \beta \\ b & \gamma & \delta\end{array}\right]$ $\left(\left|\begin{array}{ll}\alpha & \beta \\ \gamma & \delta\end{array}\right|=1\right)$ where $a=b$ or $b=0$. A particular solution is given in the following

Lemma 8.12. Let $\left(S^{4}, E^{4}, R, S\right)$ be a twin with $R$ trivial. Then, general surgery with matrix $\left[\begin{array}{lll}1 & 0 & 0 \\ d & 1 & \beta \\ 0 & 0 & 1\end{array}\right]$ gives $a$ twin in $S^{4}$.
Proof. Take $B^{3} \subset S^{4}$ such that $\partial B^{3}=R$ and $B^{3}$ is in general position with respect to $S$. Then $S \cap B^{3}$ is a disjoint union of a number of simple with respect to $S$ curves on $S$ and one arc on $S$ joining the two points of $S \cap R$. closed curves we can select on $\partial E^{4}$ the longitude $l$ such that $l \subset B^{3}$. Hence, by Hence we can select on $\partial E^{4}$ the longitude $l$ such that $T_{s} B_{s}$ is trivial, and Dehn-surgery on $T_{s}$ produces $S^{4}$ again (Corollary 5.6). As on the proof of Proposition 8.8, Dehn-surgery on $T_{s}$ with surgery data $(1, d, \beta)$ produces the same result as general surgery of matrix $\left[\begin{array}{ccc}1 & 0 & 0 \\ d & 1 & \beta \\ 0 & 0 & 1\end{array}\right]$.

Remark 8.4. When $\left(S^{4}, E^{4}, R, S\right)$ is the example a) of Section 7 , this was proved by Litherland. His proof is as follows: $\{*\} \times V^{3}$ lies in a ball $B^{3}$ such that $\partial B^{3}=R$. Then $\lambda$ extends by the identity to $B^{3}$. But then the new automorphism is isotopic to identity by Alexander trick. Finally this isotopy is realized in a neighbourhood of $B^{3}$. The new knot $S$ was called $d$-rolling, $\beta$-twist spun by Litherland.

The following Corollary generalizes Corollary 8.6 (by making $d=0$ ):

Corollary 8.13. Let $R \cup S$ be $a$ twin with $R$ and $S$ trivial. Then, general surgery with matrices

give a twin in $S^{4}$.

$$
\begin{aligned}
& {\left[\begin{array}{ccc}
1 & 0 & 0 \\
\alpha d & \alpha & \beta \\
\gamma d & \gamma & \delta
\end{array}\right], \text { where } \alpha+\gamma \text { is odd }} \\
& {\left[\begin{array}{ccc}
1 & 0 & 0 \\
\beta d & \alpha & \beta \\
\delta d & \gamma & \delta
\end{array}\right], \text { where } \beta+\delta \text { is odd }}
\end{aligned}
$$

Proof. The first case arises by the product (compare Lemma 8.11 and Remark 4.2)

$\left[\begin{array}{c|cc}1 & 0 & 0 \\ \hline 0 & P \\ 0 & \end{array}\right]\left[\begin{array}{l|ll}1 & 0 & 0 \\ \hline d & 1 & \beta \\ 0 & 0 & 1\end{array}\right]$, for $\beta=0,1$, and $P$ even.

The second case corresponds to $\left[\begin{array}{c|cc}1 & 0 & 0 \\ \hline 0 & P \\ 0 & P\end{array}\right]\left[\begin{array}{lll}1 & 0 & 0 \\ 0 & 1 & 0 \\ d & \beta & 1\end{array}\right], \beta=0,1$.

\section{Branched coverings}

Let $X^{4} /(r, s)$ be a twin in $S^{4}$. We have identified $\partial X^{4}$ with $T^{3}$ as at the beginning of Section 8 . 
The tori $T_{1}^{2}=S^{1} \times\{*\} \times S^{1}, T_{2}^{2}=S^{1} \times S^{1} \times\{*\}$ generate the kernel of $H_{2}\left(\partial X^{4} ; \mathbb{Z}\right) \stackrel{i_{*}}{\longrightarrow} H_{2}\left(X^{4} ; \mathbb{Z}\right)$, where $i_{*}$ is induced by inclusion. Let $\lambda_{1}^{\prime}$, $\lambda_{2}^{\prime} \in H^{1}\left(\partial X^{4} ; \mathbb{Z}\right)$ be Poincaré duals of $T_{1}^{2}, T_{2}^{2}$, respectively. Since $H_{2}\left(X^{4} ; \mathbb{Z}\right)$ is dual of $H^{2}(X, \partial X ; \mathbb{Z})$, the cohomology exact sequence of $(X, \partial X)$ implies that $\lambda_{1}^{\prime}, \lambda_{2}^{\prime} \in i m\left(H^{1}(X ; \mathbb{Z}) \rightarrow H^{1}(\partial X ; \mathbb{Z})\right)$. Represent $\lambda_{1}^{\prime}, \lambda_{2}^{\prime}$ by the continuous maps $\Lambda_{1}^{\prime}, \Lambda_{2}^{\prime}: \partial X \rightarrow S^{1}$ defined by $\Lambda_{1}^{\prime}(x, y, z)=y$, $\Lambda_{2}^{\prime}(x, y, z)=z$, respectively. Then we can extend $\Lambda_{1}^{\prime}, \Lambda_{2}^{\prime}$ to $\Lambda_{1}, \Lambda_{2}: X \rightarrow$ $S^{1}$, respectively.

Let $p: \tilde{X}_{m, n}^{4} \rightarrow X^{4}$ be the fiber bundle induced by $\Lambda_{1} \times \Lambda_{2}: X \rightarrow$ $S^{1} \times S^{1}$ from $(m, n): S^{1} \times S^{1} \rightarrow S^{1} \times S^{1}$. Thus $p$ is a regular covering of $m n$ sheets. We have a commutative diagram

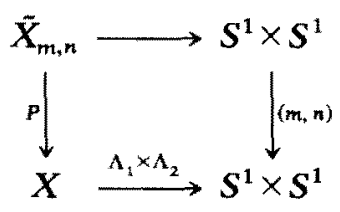

We identify $\partial \tilde{X}_{m, n}$ with $T^{3}$ by the homeomorphism $T^{3} \rightarrow \partial \tilde{X}_{m, n}$ given by $(x, y, z) \mapsto[(x, m y, n z),(y, z)]$. Then $p: \partial \tilde{X}_{m, n} \rightarrow \partial X$ is given by $(x, y, z) \rightarrow(x, m y, n z)$. Hence $p$ extends to a map $p: \tilde{X}_{m, n} /(r, s) \rightarrow X /(r, s)$, which is one to one when restricted to the twin components. We call $\tilde{X}_{m, n} /(r, s)$ the $(m, n)$-covering of $S^{4}$ branched over the twin $R \cup S$. It does not depend on the particular $\Lambda_{1}, \Lambda_{2}$. If $m$ or $n$ equals 1 we have the usual cyclic covering branched over a $2-$ knot in $S^{4}$.

Let $X^{4} /(r, s)$ be the 1-twist spun of a knot $K$ in $S^{3}$ (Example b), Section 7). Since, this twin is interchangeable, both twins $\tilde{X}_{n, 1}^{4} /(r, s), \tilde{X}_{1, n}^{4} /(r, s)$ are the $n$-twist spun twin of $A[G K]$. The $n$-twist spun knot of $A$ is $\tilde{S}$ in the first twin or $\tilde{R}$ in the second. Let us use the second twin. Here we have the restriction

$$
p^{\prime}: \tilde{X}_{1, n} /(-, s) \rightarrow X /(-, s)
$$

We identify $\partial \tilde{X}_{1, n} /(-, s), \partial X /(-, s)$ with $S^{1} \times S^{2}$ in the natural way, so that $r$ becomes the $S^{1}$-factor and the collapsed torus $T_{1}^{2}$ contains the $S^{2}$-factor. Thus $p^{\prime} \mid S^{1} \times S^{2}$ is a covering of $S^{1} \times S^{2}$ branched over $S^{1} \times S^{0}$.

The automorphism $\tau^{n}$ of $\left(S^{1} \times S^{2}, S^{1} \times S^{0}\right)$ lifts to $\tau$. Hence the twin $\tilde{X}_{1, n} /(-, s) \cup_{\tau} D^{2} \times S^{2}$ is the $(1, n)$-covering of the twin $X /(-, s) \cup_{\tau^{n}} D^{2} \times$ $S^{2}$. This twin is the $(n \pm 1)$-twist spun twin of $A$ (the sign depends on orientation; let us take the minus sign) in which the component which has meridian $s$ is the $(n-1)$-twist spun knot of $A$. Hence $X /(-, s) \cup_{\tau^{n}} D^{2} \times S^{2}$ is $\tilde{X}_{(n-1,1)} /(r, s)$. Hence we have proved

THeOREM 9.1. Let $X /(r, s)$ be the 1-twist spun twin of a knot $K$. Then,
$\left.\left(\tilde{X}_{(n-1, n)}\right)(r, s), \vec{R}\right)$ is the 2-knot which is obtained by Gluck-surgery on the $n-t w i s t$ spun knot of $K$.

The next Corollary was obtained by Litherland [G; footnote, p. 595].

COROLlary 9.2. Let $\left(S^{4}, R\right)$ be the 2-twist spun of a knot. Then $\left(S^{4}, R\right)$ equals the knot obtained by performing Gluck-surgery on $\left(S^{4}, R\right)$.

Proof. $\left(S^{4}, R\right)$ is $\left(\dot{X}_{(1,2)} /(r, s), \tilde{R}\right)$ where $X /(r, s)$ is the 1-twist spun twin of $K$. By Theorem $\left.9.1\left(\tilde{X}_{(2-1,2)}\right)(r, s), \tilde{R}\right)=R^{*}$.

\section{REFERENCES}

F. R. H. Fox, 'Rolling', Bull. Amer. Math. Soc, 72 (1966), 162-164.

F. 585-596.

CK. Kauffman, 'Twist spinning revisited', Trans. Amer. Math. Soc. $239(1978), 229-251$.

G. He embedding of two-spheres in the four-sphere', Trans. Amer. Math. H. Gluck, The embedding
Soc. 104, (1962), 308-333.

R. A. Litherland, 'Deforming twist-spun knots', Trans. Amer. Math. Soc. 250 (1979), 311-331.

L. B R Lickorish, 'A representation of orientable combinatorial 3-manifolds', Annals of Math. (2), 76 (1962), 531-540.

MII. J. Mils of (Springer Verlag) (1973).

NW. M. Newman, 'Integral matrices', vol. 45, Pure and Applied Mathematics. Academic Press (1972).

R. V. A Rohlin, 'Proof of Gudkov's conjecture', Funkt. Analiz. i ego Pril. 6, (1971) 62-64 (in Russian); English translation Funct. Anal. and its Appl. 6 (1972), 136-138. E. C. Zeeman, 'Twisting spun knots', Trans. Amer. Math. Soc. 115 (1965), 471-495.

\section{Facultad de Ciencias}

Universidad de Zaragoza

Spain 